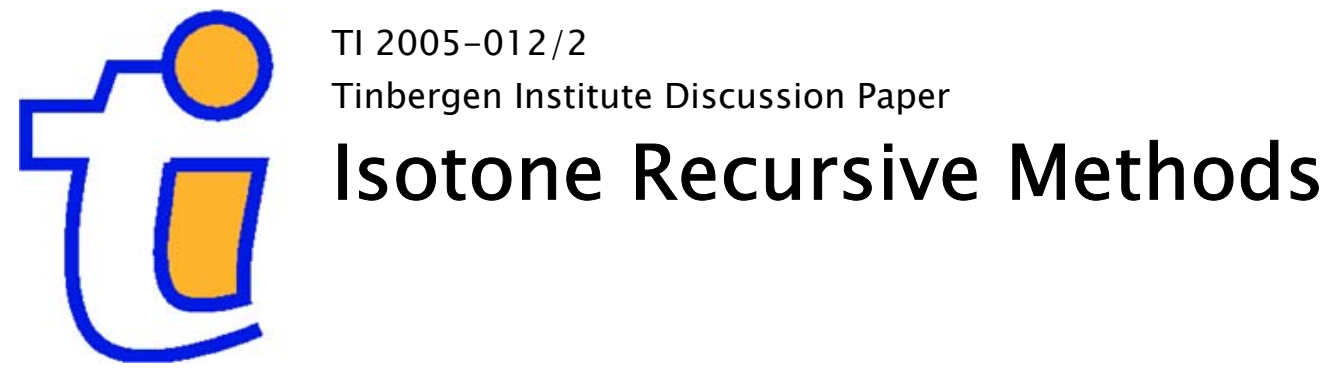

Manjira Datta

Kevin L. Reffett*

Arizona State University.

* Tinbergen Institute. 
Tinbergen Institute

The Tinbergen Institute is the institute for economic research of the Erasmus Universiteit Rotterdam, Universiteit van Amsterdam, and Vrije Universiteit Amsterdam.

Tinbergen Institute Amsterdam

Roetersstraat 31

1018 WB Amsterdam

The Netherlands

Tel.: $\quad+31(0) 205513500$

Fax: $\quad+31(0) 205513555$

Tinbergen Institute Rotterdam

Burg. Oudlaan 50

3062 PA Amsterdam

The Netherlands

Tel.: $\quad+31(0) 104088900$

Fax: $\quad+31(0) 104089031$

Please send questions and/or remarks of nonscientific nature to driessen@tinbergen.nl.

Most TI discussion papers can be downloaded at http://www.tinbergen.nl. 


\title{
Isotone Recursive Methods: The Case of Homogeneous Agents*
}

\author{
Manjira Datta \\ Arizona State University
}

\author{
Kevin L. Reffett \\ Arizona State University
}

January 2005

\begin{abstract}
Over the last decade, isotone recursive methods have provided unified catalog of results on existence, characterization, and computation of Markovian Equilibrium Decision Processes (MEDPs) in infinite horizon economies where the second welfare theorem fails. Such economies include models with production nonconvexities, taxes, valued fiat money, models with monopolistic competition, behavioral heterogeneity, and incomplete markets. In this paper, we survey this emerging class of methods. Our methods use a qualitative approach to economic equilibria first introduced in the work in operations research by Veinott and Topkis. As the methods emphasize the role of order, they are amenable for obtaining conditions for monotone comparison theorems on the space of economies. We are also able to describe monotone iterative procedures that provide the needed foundations for a theory of numerical solutions for MEDPs and stationary Markov equilibrium (SME). One interesting additional result of independent interest is we construct sufficient conditions for the existence of a new class of envelope theorems for nonconcave programming problems.
\end{abstract}

*Email addresses: Manjira.Datta@asu.edu, Kevin.Reffett@asu.edu. We are deeply indebted to Len Mirman and Olivier Morand for numerous lengthy discussions concerning many issues discussed in this survey. Many of the results presented in this paper were developed originally in some form during our joint work with Len and Olivier over the last five years. We dedicate this paper to Len Mirman on the occasion of his sixty-fifth birthday. Indeed, this paper would not have been written without Len's ongoing pioneering work on equilibrium growth under uncertainty. We also thank Elena Antoniadou, Hector Chade, John Coleman, Jeremy Greenwood, Seppo Heikkila, Ken Judd, Tom Krebs, Cuong Le Van, Robert Lucas, Jr., Jianjun Miao, Chris Shannon, John Stachurski, Yiannis Vailakis, Charles Van Marrewijk, Jean-Marie Viaene, Itzhak Zilcha, and especially Robert Becker and Manuel Santos for many helpful conversations over the past years. All mistakes remain our own. 


\section{Introduction}

A foundation of modern macroeconomics is the stochastic growth model originally introduced in the seminal work of Brock and Mirman[15]. Their original model is an infinite horizon economy with a continuum of identical households, each with access to a complete set of financial markets that insure them against all sources of idiosyncratic risk. There is single sector production that employs capital and labor whose returns are summarized by a stochastic neoclassical production function representing an aggregate convex production set with identical private and social returns to inputs. There is also aggregate risk taking the form of a collection of identically and independently distributed (i.i.d.) random variables, the agents in the economy face no frictions in information acquisition (i.e., there is no learning), labor supply is inelastic, and there are no equilibrium distortions. The authors characterize the unique Markovian Equilibrium Decision Process (MEDP) and its associated unique (non-trivial) long-run equilibrium dynamics, in particular, the Stationary Markovian Equilibrium (SME). Their methodological approach was pioneering, and relied heavily on recursive methods. Implicitly, it exploits the validity of a second welfare theorem and one can interpret the economic outcomes of the fictional social planner's problem from the perspective of a decentralized economic system. A fully decentralized recursive formulation of the Brock-Mirman framework is put forward by Prescott and Mehra[61] (see also, Stokey, Lucas, with Prescott[73]).

Over the last three decades, extensions of this model have become the foundation for the systematic study of many diverse issues in quantitative dynamic macroeconomic theory. Applications include models of economic fluctuations and business cycles, production-based asset pricing, the positive and normative implications of incomplete financial markets and public goods, the wealth inequality, the dynamic structure of altruistic economies, stochastic life-cycle models, models with physical and human capital, and the role of activist fiscal and/or monetary policy etc. However, many recent applications emphasize economic environments where the second welfare theorem is not available. These modifications create serious complications for a systematic study of the underlying structure of the MEDPs and the SME. A prevalent approach is to develop extensive applications of numerical methods to characterize MEDPs and the SME. From a mathematical perspective, many of these approaches have been ad hoc as they cannot be developed rigorously without providing characterizations of qualitative structure of the MEDPs and/or the SME.

An important question naturally emerges from this apparent disconnect 
between mathematical principle and macroeconomic practice: can one provide sharp and constructive characterizations of the MEDPs or the SME for generalized Brock-Mirman environments where the second welfare theorem fails? The most significant advance in providing an affirmative answer to this question has been the recent literature on "monotone methods" (also known as "monotone map" methods or "isotone recursive methods"). The pioneering work of Coleman [18][19][20][21], Greenwood and Huffman[34], Datta, Mirman, and Reffett[22] and Morand and Reffett[57] provide the genesis of the study of isotone recursive methods over the last fifteen years (they refer to them, as the "monotone-map" method). These papers present the first set of conditions under which constructive methods can be applied for studying the structure of a decentralized Markovian equilibrium in economies with or without non-classical production technologies. ${ }^{1}$ An important generalization of this monotone-map approach is found in Mirman, Morand, and Reffett[54]. Here, a new and more general isotone map approach is presented (with the Coleman-Greenwood-Huffman approach as a special case) and can be applied to a larger collection of dynamic economies with production nonconvexities (in the reduced-form production function). In this setting, sets of sufficient conditions for the existence of semicontinuous, continuous, Lipschitz continuous, and once-differentiable MEDPs are given. Since sufficient conditions for MEDPs to be differentiable are presented, therefore the error bounds constructed in Santos and Vigo[70] and Santos[69] apply. Finally a theory of ordered MEDPs is developed applying the seminal work in operations research on lattice programming and the qualitative study of equilibrium introduced in Veinott[80][81] and Topkis[76][77][78].

The chapter is organized as follows: in the next section, we introduce some useful terminology. Section 3 provides a survey of the existing literature on fixed point theory in order spaces. This fixed point theory is critical in the development of isotone recursive methods. In Section 4, we consider homogeneous agent economies with classical production technology and infinite horizon. In this section, we develop an "Euler equation" approach to isotone recursive methods. We discuss the case studied in Coleman[19] for nonoptimal homogeneous agent economies. In Section 5, we discuss the generalizations found in Mirman, Morand, and Reffett[54]. Section 6 considers

\footnotetext{
${ }^{1}$ The literature on monotone map methods is vast, and also includes the papers of Lucas and Stokey [51], Bizer and Judd [14] etc. An interesting alternative monotone method is developed in Becker and Foias [9].

For non-existence of a continuous MEDP, see Santos [68] and Krebs [45]. Mirman, Morand, and Reffett ([54], section 4) show that although the Santos [68] example is robust to a large class of economies, in many case MEDPs are semi-continuous and isotone.
} 
the case of elastic labor supply as in Coleman[20] and Datta, Mirman and Reffett[22]. In section 7, we conclude with a brief discussion of new frontiers in monotone recursive methods, to models with heterogeneous agents including the overlapping generations models with stochastic production (e.g., Erikson, Morand and Reffett[31]), models with unbounded stochastic nonoptimal growth (e.g., Morand and Reffett[57]), Ramsey-type models with heterogeneous agents (e.g., Datta, Mirman, Morand and Reffett[23], and the mixed monotone recursive methods discussed in Reffett[63] and Mirman, Reffett, and Stachurski[55].

\section{Preliminaries}

\subsection{Ordered Spaces}

We begin with some useful terminology. For a more complete accounting of the ideas in this section, see Birkhoff[13], Veinott[81], and Davey and Priestley[24].

Qosets and Posets: Let $X$ be a set. We say $X$ is quasi ordered (or a qoset) if $X$ is equipped with an order relation $\geq_{X}: X \times X \rightarrow X$ that is reflexive and transitive. In our subsequent discussion, we shall respect two notational conventions: (i) we write " $\geq$ " in place of " $\geq_{X}$ " when the order relation $\geq_{X}: X \times X \rightarrow X$ is clearly implied; and (ii) for two elements of an qoset $X$, say $a$ and $b$, the order relation " $a \geq b$ " can also be written as " $b \leq a$ ". If every element of as qoset $X$ is comparable, we say $X$ is a complete qoset.

The set $X$ is referred to as a partially ordered set (or poset or simply an ordered set) if $X$ is equipped with an order relation $\geq$ that is reflexive, transitive, and antisymmetric. If every element of a poset $X$ is comparable, then we say $X$ is a totally ordered set or chain. As we shall see in the immediate sequel, every chain has an inherent lattice structure.

Lattices. Let $X$ be a poset equipped with a partial order $\geq$. An upper (respectively, lower) bound for a set $B \subset X$ is an element $x^{u}$ (respectively, $\left.x^{l}\right) \in B$ such that for any other element $x \in B, x \leq x^{u}$ (respectively, $x^{l} \leq x$ ) for all $x \in B$. If there is a point $x^{u}$ (respectively, $\mathrm{x}^{l}$ ) such that $x^{u}$ is the least element in the subset of upper bounds of $B \subset X$ (respectively, the greatest element in the subset of lower bounds of $B \subset X$ ), we say $x^{u}$ (respectively, $x^{;}$) is the supremum (respectively, infimum) of $B$. Clearly if they exist, both the supremum (or, sup) and infimum (or, inf) must be unique. We say $X$ is a lattice if for any two elements $x$ and $x^{\prime}$ in $X, X$ is closed under the operation of infimum in $X$, denoted $x \wedge x^{\prime}$, and supremum in $X$, denoted 
$x \vee x^{\prime}$.The former is referred to as "the meet", while the latter is referred to as "the join" of the two points, $x, x^{\prime} \in X$. A subset $B$ of $X$ is a sublattice of $X$ if it contains the sup and the inf (with respect to $X$ ) of any pair of points in $B$. A lattice is complete if any subset $B$ of $X$ has a least upper bound $\vee B$ and a greatest lower bound $\wedge B$ in $B$. If every chain $C \subset X$ is complete, then $X$ is referred to as a chain complete poset (or equivalent, a complete partially ordered set or $C P O)$. A set $C$ is countable if it is either finite or there is a bijection from the natural numbers onto $C$. If every chain $C \subset X$ is countable and complete, then $X$ is referred to as a countably chain complete poset. Finally, a subset $A$ of a set $C \subset P$ is cofinal if for each $x \in C$, there is a $y \in A$ such that $x \leq y$.

Ordered vector spaces and cones. A partially ordered vector space or linear semi-ordered space is a poset $X$ that is real vector space equipped with a partial order $\geq$ that is compatible with the following algebraic structure: (i) if $x \geq x^{\prime}$, then $x+z \geq x^{\prime}+z$, for all $z \in X$; (ii) if $x \geq x^{\prime}$, then $\alpha x \geq \alpha x^{\prime}$ for all $\alpha \geq 0$. Any partially ordered vector space that is also a lattice is called a vector lattice. If the space has a norm $\|x\|_{X}$ which satisfies whenever $|x| \geq\left|x^{\prime}\right|$ in $X,\|x\| \geq\left\|x^{\prime}\right\|$, we say $X$ has a lattice norm. A complete normed vector space is a Banach space. A normed vector lattice is a vector lattice equipped with a lattice norm. A normed vector lattice $X$ that is complete in the Cauchy sense, and is endowed with a lattice norm is referred to as a Banach lattice.

Let $X$ be a topological space. The set $X^{+}=\{x \in X, x \geq 0\}$ is the order cone of $X$ if $X$ is nonempty convex closed set that has the following two properties: (i) $x \in X^{+} \Longrightarrow \alpha x \in X^{+}$for $\alpha \geq 0$; (ii) if $x$ and $-x$ in $X^{+}, x=0$ where 0 denote the zero of the cone. The partial order induced by the cone structure of $X^{+}$has $x_{1} \geq x_{2}$ if $x_{1}-x_{2} \in X^{+}$. Now, assume $X$ is a real Banach space. A cone $X^{+}$of $X$ is normal if there exists a constant $m$ such that for any $x_{1}, x_{2} \in X^{+},\left\|x_{1}+x_{2}\right\| \geq m,\left\|x_{i}\right\|=1$ for $i=1,2$. Intuitively, the restriction of normality of the cone geometrically bounds the angle between any two unit vectors away from $\pi$, so a normal cone cannot become "too large". An increasing sequence in the cone $\left\{x_{t}\right\}_{t=1}^{t=\infty}, x_{t} \in X^{+}$ is a sequence that satisfies $x_{1} \leq x_{2} \leq \ldots \leq x_{n} \leq \ldots$ We say a cone $X^{+}$is regular if if every increasing and bounded order sequence in $X^{+}$has a limit in $X^{+}$. We say $X^{+}$is fully regular if every increasing and norm bounded sequence in $X^{+}$has a limit in $X^{+}$. A fully regular cone is also regular. A regular cone is normal. (See Guo and Lakshmikantham[35], Theorem 1.2.1). A cone $X^{+}$is solid if its interior $\dot{X}^{+}$is nonempty.

Let $[a)=\{x \mid x \in X, x \geq a\}$ be the upperset of $a,(b]=\{x \mid x \in X, x \leq b\}$ the lowerset of $b . X$ is an ordered topological space if $X$ is equipped with a 
partial order and topology that implies $[a)$ and $(b]$ are closed in the topology on $X$. An order interval is defined to be $[a, b]=[a) \cap(b], a \leq b$. Therefore in an ordered topological space, all $[a, b] \subset X$ (e.g., order intervals) are closed in the topology of $X$. In our work, we will often study fixed point problems where the domain/range is a compact order interval in a normal and solid cone of positive continuous functions $X^{+}=C^{+}(S)$ endowed with the $C^{0}$ uniform norm topology (where each function itself is defined on compactum $S$ ). Such a space is not a regular cone. We will often work on a transformation space that is a compact suborder interval in $C^{+}(S)$ (where compactness will be used to compensate for the loss of regularity in the cone $\left.C^{+}(S)\right)$.

\subsection{Mappings}

We now define some important properties of mappings, especially those defined on lattices and posets:

Isotone (or Order Preserving) Mappings on a Poset: Let $\left(X, \geq_{X}\right)$ and $\left(Y, \geq_{Y}\right)$ be Posets. A mapping is a relational statement between two spaces, say $X$ and $Y$. We shall consider both "point-to-point" and "pointto-set" mappings. In the case of a "point-to-point" mapping, we refer to the mapping as a function (or equivalently as an operator). A function $m: X \rightarrow Y$ is said to be isotone on $X$ if it is "order-preserving", i.e., $m\left(x^{\prime}\right) \geq_{Y} m(x)$, when $x^{\prime} \geq_{X} x$, for $x, x^{\prime} \in X$. If $m\left(x^{\prime}\right)>_{Y} m(x)$ when $x^{\prime}>_{X} x$ for $x, x^{\prime} \in X$, we say the function $m$ is increasing. If $m\left(x^{\prime}\right)>_{Y} m(x)$ when $x^{\prime} \geq_{X} x, x^{\prime} \neq x$, we say the function $m$ is strictly increasing. We say $m(x)$ is antitone (or, order-reversing) if $m(x) \geq_{Y} m\left(x^{\prime}\right)$ if $x^{\prime} \geq_{x} x$. A function that is either isotone or antitone is monotone. When the mapping $m(x)$ is a self-mapping on $X$, we also refer to $m(x)$ as a transformation of $X$, and the set $X$ as a transformation set. If our concern is the fixed points of a transformation $m(x)$ on $X$, we refer to the transformation set $X$ as the fixed point space.

Notions of monotonicity are also available for multifunctions or correspondences. By a correspondence or multifunction, we always refer to a nonempty-valued mapping $M: X \rightarrow 2^{Y}$, e.g., a nonempty-valued "pointto-set" mapping. We say a correspondence or multifunction is ascending in the set relation $S$ (denoted by $\geq_{S}$ ) if $M\left(x^{\prime}\right) \geq_{S} M(x)$, when $x^{\prime} \geq_{X} x$ where $\left(X, \geq_{X}\right)$ is a partially ordered space. If this set relation $\geq_{S}$ induces a partial order on the powerset $2^{Y}$ (or, perhaps, $2^{Y} \backslash \emptyset$ ), we refer the ascending correspondence also as an isotone correspondence.

To make concrete the notion of an isotone versus ascending correspon- 
dence, we discuss some particular set relations; some that induce partial orders on $2^{Y}$ (or, $2^{Y} \backslash \varnothing$ ), others that do not. ${ }^{2}$ The set relations we consider are each compatible with pointwise set comparisons, and, therefore, closely related to the sufficient conditions under which correspondences admit isotone selections. We focus primarily on four such set relations. Let $Y$ be a set, and $A, B \in 2^{Y}$. We define : (i) the Veinott-Weak Set relation $\geq_{w}$ on $2^{Y} \backslash \varnothing: A \geq_{w} B$, if for any $a \in A, b \in B$, either $a \wedge b \in B$, or, $a \vee b \in A$; (ii) the Veinott-Strong Set Order $\geq_{s}$ on $2^{Y} \backslash \varnothing: A \geq_{a} B$, if for any $a \in A$, $b \in B, a \wedge b \in B$ and $a \vee b \in A$; (iii) the Smithson-Weak Set relation $\geq_{a s}$ on $2^{Y}: A \geq_{a s} B$ if we have either (C1) for any $b \in B$, there exists an $a \in A$ such that $a \geq b$; or, (C2) for any $a \in A$, there exists an $b \in B$ such that $a \geq b$; (iv) the Pointwise Strong Set Order $\geq_{s s}$ on $2^{Y} \backslash \varnothing: A \geq_{s s} B$ if and only if $a \in A, b \in B$, then $a \geq b$ in the partial order structure on $A$, for all $a, b$.A final classic partial order on the powerset $2^{Y}$ is commonly referred to as set inclusion. We say a subset $A \geq_{S I} B$ under set inclusion $\geq_{S I}$ if $B \subset A$.

Fixed points. Let $\mu: X \rightarrow 2^{X}$ be a non-empty valued correspondence for each $x \in X$. The correspondence $\mu$ is said to have a fixed point if there exists an $x$ such that $x \in \mu(x)$. Therefore, if $\mu$ is a function, then a fixed point is an $x^{*}$ such that $x^{*}=\mu\left(x^{*}\right)$. A fixed point $x^{*}$ is minimal (respectively, maximal) if there does not exist another fixed point, say $y^{*}$, such that $y^{*} \leq x^{*}$ (respectively, $x^{*} \leq y^{*}$ ). If a fixed point is either minimal or maximal, we say it is extremal.

\section{$3 \quad$ Fixed Point Theory In Ordered Spaces}

In this section, we provide an account of fixed point theory in ordered spaces. For a more extensive discussion, see excellent surveys in Amann[4], Guo and Lakshmikantham[35], Heikkila and Lakshmikantham[36] and Jachymski[40].

\subsection{Existence}

First, we discuss the existence and characterization of solutions for two prototypical classes of parameterized fixed point (or, transformation) problems often encountered in economic applications. Consider $X$ is a poset, $T$ is an ordered topological space. The two problems are stated as Problem 1 and Problem 2.

\footnotetext{
${ }^{2}$ For a more detailed discussion, we refer to the classic references of Smithson [72] and Veinott [81]
} 
Problem 1: To characterize the fixed points of the mapping,

$$
f(x, t): X \times T \rightarrow X \text { and } f \text { is isotone on } X \text { for each } t \in T \text {. }
$$

Problem 2: To characterize the fixed points of the mapping,

$F(x, t): X \times T \rightarrow 2^{X}, F$ is ascending $\left(\geq_{a s}\right)$ in $(\mathrm{C} 1)$ or $(\mathrm{C} 2)$ on $X$ for each $t \in T$. $2^{X}$.

Recall that $\geq_{a s}$ denotes Smithson's weak set relation on the powerset

\subsubsection{Lattice Theoretic Fixed Point Theorems}

A classical case of Problem 1 occurs when $X$ is a nonempty, complete lattice. This is the case studied in the seminal work of Tarski[74],[75] in the early 1940s, see also Kantorovich[43]. ${ }^{3}$ We say a space $Y$ has a fixed point property for isotone functions (or, more compactly, fpp) if and only if each isotone transformation of $Y$, say $f: Y \rightarrow Y$, has a fixed point. We denote the fixed point correspondence, in either Problem 1 or Problem 2, as $G(t)$.

We state Tarski's theorem adapted to Problem 1:

Proposition 1 (Tarski[75], Theorem 1): Fix $t \in T$, and let $f(x, t): X \times$ $T \rightarrow X, f$ be isotone in $x$ for each $t \in T$. Then $G(t)$ is an nonempty complete lattice for each $t \in T$.

We make a few remarks on this result. First, the theorem does not say $G(t)$ is subcomplete in $X$. In general, it is not. Second, the operator $f$ is assumed to have no continuity properties on $X$ (e.g., we assume no order or topological continuity properties for $f(x, t))$.

Often in economic applications, because of the absence of sufficient concavity in the agent's decision problem along equilibrium trajectories, equilibrium fixed point problems cannot be posed in terms of a single valued operator such as in Problem 1; rather, they must be posed in a more abstract setting of the fixed point of multifunctions, as in Problem 2. For the general case, a key generalization of Tarski was obtained by Veinott[80] in the 1970s, see also Veinott[81] (Chapter 4, Theorem 14). ${ }^{4}$

\footnotetext{
${ }^{3}$ Tarski's original result dates from around 1942 and is available in Tarski [74]. It is a generalization of a result he developed with Knaster in 1921 (for isotone correspondences under set inclusion). A related result for semi-ordered linear spaces is in Kantorovich [43].

${ }^{4}$ Zhou [83] proves it independently in Theorem 1.
} 
Proposition 2 (Veinott[81]):Let $F(x, t): X \times T \rightarrow 2^{X} \backslash \varnothing$. For any fixed $t \in T$, assume that $F(x, t)$ is a nonempty, isotone in Veinott's strong set order, closed, and sublattice-valued correspondence on $X$. If $G(t)$ is the fixed point correspondence for $F(x, t)$ at $t \in T$, then $G(t)$ is a nonempty complete lattice for each $t \in T$.

Propositions 1 and 2 provide sufficient conditions for the existence of a complete lattice of fixed points for an isotone and/or ascending transformations of a complete lattice $X$. An interesting question is necessity: i.e., can one obtain a complete characterization of a complete lattice using the fixed point property? Davis[25] (Theorem 1) provides the converse to Tarski's theorem: a lattice $X$ is complete if and only if every isotone transformation $f: X \rightarrow X$ has a fixed point. In the context of Problem 2, the Davis characterization of a complete lattice $X$ is also provided. Smithson[72] (corollary 1.8) proves the following: if $X$ is a lattice and $F(x)$ is a multifunction then $X$ is complete if and only if the correspondence $F(x)$ is (a) ascending in the Smithson-weak set relation (C1) (respectively, ascending in the Smithsonweak set relation (C2)), and (b) the least upper bound $(F(x, t)) \in F(x, t)$ (the greatest lower bound $(F(x, t)) \in F(x, t))$ for all $x \in X, t \in T$, and $G(t)$ is nonempty for each $t \in T$.

Other useful characterizations of complete lattices are available, and we use them in the sequel, as needed. For example, one can characterize a complete lattice $X$ in terms of its interval topology (Frink[32]). Recall, the interval topology for a set $X$ takes all the closed intervals $[a, b]$ as a subbasis for the closed sets of $X$. Frink[32] provides the following characterization of a complete lattice $X: X$ is a complete lattice if and only if $X$ is compact in its interval topology (see also Birkhoff [13], Chapter 10, Theorem 20). Another very useful characterization of a complete lattice is in Davey and Priestly[24] (Theorem 2.31). Their result provides the following characterization of a complete lattice $X$ : let $X$ be a nonempty ordered set; then the following statements are equivalent (i) $X$ is a complete lattice; (ii) for any subset $S \subset X, \inf (S) \in X$; and $X$ has a top element and $\inf (S) \in X$ for every nonempty subset of $X$. These two characterizations of a complete lattice $X$ are used repeatedly in this chapter.

\subsubsection{Fixed Point Theory in Complete Partially Ordered Sets}

Next, we now consider Problems 1 and 2 when the fixed point space $X$ is not a complete lattice. A natural set of regularity conditions for an ordered set $X$ to have the fixed point property turns out to be chain-completeness. 
Recall a set $X$ is chain complete if for any chain $C, \inf (C)$ and $\sup (C)$ are in $X$. A set $X$ has a bottom element a (respectively, top element $b$ ) if for every $x \in X, a \leq x$ (respectively, $x \leq b$ ). A set $X$ is a complete partially ordered set (or, $C P O$ ) if and only if (i) $X$ has a bottom element, and (ii) for each directed net $D \subset X$, we have a sup $D \in X$. A set $X$ is a CPO if and only if every chain $C$ in $X$ has a least upper bound, $\sup (C) \in X$. (Davey and Priestley[24], Theorem 8.11). Therefore, the notion of a set $X$ being "chain-complete" is equivalent to the space $X$ being a CP0. We often use this terminology when discussing chain-completeness.

Chain completeness is a natural condition to check in applications. For example, every relatively compact chain $C$ in an ordered topological space has an infimum and a supremum, $\inf (C)$ and $\sup (C)$. See Amann[4], Lemma 3.1. Therefore, every compact ordered topological space is chain complete (Amann[4], Corollary 3.2). One of the earliest results on the existence of a fixed point for a self map on a poset is obtained in Bourbaki[16]. As a consequence of Zorn's lemma, it is shown that if $X$ is an ordered set such that every chain has an upper bound (respectively, a lower bound), and $f(x)$ on $X$ is increasing in the following sense: for all $x \in X, x \leq f(x)$ (respectively, $f(x) \leq x)$, then $f$ has at least one fixed point. An improvement on this result is given in Abian and Brown[1] (Theorems 2,3,4) and Pelczar[60]. The version of the theorem that we state is due to Amann[4] (see also Zeidler [84], Section 11.9 for a proof):

Proposition 3 (Amann[4], Theorem 1.4): Let $X$ be a CPO, $f(x, t): X \times$ $T \rightarrow X$ be isotone in $X$ for each $t \in T$. Suppose there exists a pair $\left(x_{L}, x^{U}\right) \in$ $X \times X, x_{L} \leq x^{U}$ such that $x_{L} \leq f\left(x_{L}\right)$ and $f\left(x^{U}\right) \leq x^{U}$. Then $f$ has a minimal and a maximal fixed point in $\left[x_{L}, x^{U}\right]$.

We next consider a converse to this theorem. ${ }^{5}$ That is, as in the case of a complete lattice, we ask if one can obtain a characterization of a CPO $X$ using the fixed point property relative to isotone transformations. Clearly, an arbitrary ordered set $X$ does not have a fixed point property; but it turns out that if $X$ is an ordered set, and for each isotone operator $f(x)$ on $X$, $f(x)$ has a least fixed point, then $X$ is a CPO. Alternatively, if $G$ is the set of fixed points of an isotone self-map $f(x)$, and $X$ is a CPO, then $G$ is a CPO.

\footnotetext{
${ }^{5}$ An important converse to the Bourbaki fixed point principle (also due to Zermelo) is related to the fixed point result in the Abian-Brown-Pelczar theorem is in Jachymski[41].
} 
Proposition 4 (Davey and Priestley[24], Propositions 8.25, 8.26). Let $X$ and fix $t \in T$. Then we have the following: (i) if every isotone map in $X, f(x, t): X \times T \rightarrow X$, has a minimal fixed point $x^{*}(t)$, then $X$ is a CPO; (ii) if $f(x, t): X \rightarrow X$ is isotone on $X$ for each $t \in T$, and $G(t)$ denotes the set of fixed points of $f(x, t)$ at $t$; then if $X$ is a CPO, $G(t)$ is a CPO.

Next, we discuss generalizations of Proposition 3 to the case of multifunctions. The seminal references are Smithson[72] and Muenzenberger and Smithson[59]. Let $\mathbf{X}$ and $\mathbf{Y}$ be CPOs, $F(x): \mathbf{X} \rightarrow \mathbf{2}^{\mathbf{Y}} \backslash \varnothing$ be a nonempty correspondence, and $X \subset \mathbf{X}$ a subchain. If for any isotone function $f(x): X \rightarrow \mathbf{Y}$ such that $f(x) \in F(x)$, for $x_{0}=\sup X$, we have $f\left(x_{0}\right) \leq y\left(x_{0}\right) \in F\left(x_{0}\right)$, we say the mapping $F(x)$ has the property of Majorizing Chain Subcompleteness (MCSC). For correspondences that are ascending in Smithson's weak set relation (C1) or (C2), and that satisfy MCSC, we have the following generalization of Amann[4]:

Proposition 5 (Smithson[72], Theorem 1.1): Let $X$ be a CPO, and suppose $F(x, t)$ is isotone in the Smithson-weak set relation, (C1) and/or (C2), and satisfies Condition MCSC. If there is a point $x_{L} \in X$ and a point $y \in F\left(x_{L}\right)$ such that $x_{L} \leq y$, then $F(x, t)$ has a fixed point for each $t$.

Note that Smithson[72] (Proposition 1.6) obtains a generalization of Abian and Brown's[1] fixed point theorem for the case the $X$ is a CPO. In recent work, Heikkila and $\mathrm{Hu}$ [38] and Heikkila and Reffett[37] have generalized it further.

\subsection{Computational Fixed Point Theory}

Recall that an operator $f(x): X \rightarrow Y$ is order-continuous if for any countable chain $\left\{x_{n}\right\}$ having a supremum, we have $\sup f\left(x_{n}\right)=f\left(\sup x_{n}\right)$. If operators are order-continuous in Problem 1, we can weaken the conditions on the fixed point space $X$, and also obtain stronger results on computing extremal fixed points by successive approximation on an operator from lower solutions $x_{L}$ (e.g., a point $x_{L}$ that has $x_{L} \leq f\left(x_{L}\right)$ and upper solutions $x_{U}$ (e.g., a point $x_{U}$ that has $f\left(x_{U}\right) \leq x_{U}$ ). The successive approximations indexed on the natural numbers can be shown to converge to extremal fixed points. If the underlying space is an ordered metric space, numerical implementations of our methods via Krasnoselskii et al[44] (Chapter 4) can be shown to provide a posteriori error bounds in the underlying metric on $X$. 
This is particularly useful in our work, as many of the fixed point spaces we use have uniform metric topologies (the economies studied in Sections 4-6).

We next discuss a result due to Kantorovich[43]. This result is available in a number of places in the literature (e.g., Dugundji and Granas[30] Theorem 4.2, Vulikh[82] Theorem XII.2.1, and Davey and Priestley[24] Theorem 8.15). We have the following result for a special case of Problem 1:

Proposition 6 (Kantorovich[43]): Let $X$ be a poset, $D=[a, b] \subset X$ countably chain complete. Assume for each $t \in T, f(x, t): X \times T \rightarrow X$ is order continuous in $x$, such that $a \leq f(a, t)$ and $f(b, t) \leq b$. Let $G(t)$ be the fixed point correspondence of $f(x, t)$ for $t \in T$.Then $(i) G(t)$ is nonempty, and (ii) $\lim _{n} f^{n}(a ; t) \rightarrow \inf G(t)$ (respectively, $\lim _{n} f^{n}(b ; t) \rightarrow \sup G(t)$ ).

An alternative setting that is common in economic applications of Problem 1 has the following structure: (i) the domain $D \subset X$ is a compact order interval in a normal cone of positive continuous functions $C(X)$, where $X \subset R^{n}$ is also compact, and (ii) the operator $f(x, t)$ continuous and compact (e.g., completely continuous) in $x$ for each $t \in T$. This is true in case of Coleman[19] and Datta et al[22] for the fixed point problem that constructs MEDPs. In this case, one can apply an important theorem due to Amann[3]:

Proposition 7 (Amann[3], Theorem 6.1; corollary 6.2): Let $X$ be an ordered Banach space, $\left[x_{L}, x^{U}\right]$ an order interval with $x_{L}, x^{U} \in X, x_{L} \leq x^{U}$, $f(x, t): X \times T \rightarrow X$ is isotone on $\left[x_{L}, x^{U}\right]$, compact and continuous in $x$, such that for each $t, x_{L} \leq f\left(x_{L}\right)$ and $f\left(x^{U}\right) \leq x^{U}$. Let $G(t)$ be the set of fixed points of $f(x, t)$ at $t \in T$. Then (i) $G(t)$ is nonempty for each $t \in T$; (ii) $\lim _{n \rightarrow \infty} f\left(x_{L} ; t\right) \rightarrow \inf G(t)$ and $f\left(x^{U}, t\right) \rightarrow \sup G(t)$ and the sequences $\left\{f_{n}\left(x_{L}, t\right)\right\}_{n=0}^{t=\infty}$ and $\left\{f_{n}\left(x^{U}, t\right)\right\}_{n=0}^{n=\infty}$ are increasing and decreasing sequences, respectively.

For both propositions above, it is important that we obtain sufficient conditions that allow one to tie directly the computation of extremal fixed points to well-known numerical approximation algorithms in the existing literature (e.g., Krasnoselskii et al[44] and Judd[42]). In some cases, such indexation on the natural number are not sufficient to show that successive approximation from lower or upper solutions for a particular set of fixed points actually computes an extremal fixed point. See the example in Davey and Priestley[24], section 8.16 or Heikkila and Lakshmikantham[36], example 
1.1.1. In such cases, one can still define iterations on well-defined index sets that are subsets of chains. Heikkila and Lakshmikantham [36] address this issue and deliver a generalized iterative method on a chain. A critical advantage of their approach is that it does not require either the axiom schema of replacement or the axiom of choice.

Proposition 8 (Heikkila and Lakshmikantham[36], lemma 1.1.1): Let D be the set of subsets of $P, P$ a poset with $\varnothing \in D$ and $f: D \rightarrow P$, there is a unique well-ordered chain $C$ so that $x \in C$ if and only if $x=f\{y \in C \mid y<x\}$. If $f(C)$ exists, it is not a strict upper bound of $C$.

We discuss the elements in the chain C.Standard transfinite iterations are contained: let $x_{0}=f(\varnothing), x_{n+1}=f\left(\left\{x_{0}, x_{1}, \ldots, x_{n}\right\}\right)$ for $x_{n}<x_{n+1}$, , $x_{w}=f\left(\left\{x_{n}\right\}_{n=0}^{n=\infty}\right)$ with $x_{w}$ a strict upper bound of $\left\{x_{n}\right\}_{n=0}^{n=\infty}$, then $x_{w}$ is a next successor element of $C$, and so forth. When establishing conditions in applications under which the generalized iterations of the mapping $f$ can be indexed on countable sets, it is useful to recall that by Zorn's lemma, if each well-ordered chain $C$ in $P$ has an upper bound in $P$, then $P$ has a maximal element. From Heikkila and Lakshmikantham[36], Lemma 1.1.2, we know that each chain of any poset contains a well-ordered cofinal chain. Further, by another lemma in Heikkila and Lakshmikantham[36] Lemma 1.1.4, a wellordered chain $C$ in a poset $P$ is countable if its subchains possess countable cofinal chains. Finally, a monotone sequence in an ordered topological space $X$ converges if each of its subsequences has a cluster point. A natural question concerns sufficient conditions under which iterations on $f$ from some lower solution $x_{L}$ converge to fixed points on a countable indexation of iterations. One set of sufficient conditions are as follows:

Proposition 9 Heikkila and Lakshmikantham[36], Lemma 1.1.7; Proposition 1.1.5; Proposition 1.1.6: (i) If a chain $C$ in an ordered topological space $X$ has a separable cofinal subset $A$, and if each nondecreasing sequence of $A$ has a cluster point in $X$, then $C$ contains a nondecreasing sequence that converges to $\sup C$; (ii) a well ordered chain of $X$ is countable if the following occurs: (a) $X$ is first countable, and each subchain of $C$ is relatively compact; (b) each subset of $C$ is separable and each nondecreasing sequence of $C$ has a cluster point; (iii) If $C$ is a chain in an ordered metric space $X$, and if each nondecreasing sequence of $C$ has a cluster point, then $C$ contains a nondecreasing sequence which converges to $\sup C$, and $C$ is countable if each nondecreasing sequence of $C$ has a cluster point. 


\subsection{Monotone Selections and the Equilibrium Correspon- dence}

In Problem 1 and Problem 2, a natural question to analyze is the existence of monotone comparison theorems on the space of parameters $T .{ }^{6}$ Let $G(t)$ : $T \rightarrow 2^{X} \backslash \varnothing$ denote the fixed point correspondence. We say the fixed point Problem 1 or 2 exhibits a strong comparative structure (SCS) if the fixed point correspondence $G(t)$ is an isotone correspondence from $T \rightarrow 2^{X} \backslash \varnothing$. We say Problem 1 or 2 exhibits a weak comparative structure (WCS) if its fixed point correspondence $G(t)$ admits an isotone selection. First, consider the SCS. Known sufficient conditions for $G(t)$ to be consistent with SCS involve the fixed point space $X$ be a complete lattice, ordering the range of $G(t)$ using Veinott's strong set order on $2^{X} \backslash \varnothing$, and proving that $G(t)$ has a sublattice structure in $2^{X} \backslash \varnothing$. For example, if $G(t)$ is isotone from $T$ to $2^{X} \backslash \varnothing$ in Veinott's strong set order, one immediately has the extremal selections $\sup G(t)$ and $\inf G(t)$ as isotone operators on $T$. The most general version of the result we discuss is due to Veinott[81] (Chapter 4, Theorem 14) and Topkis[78] (Theorem 2.5.2). The Veinott-Topkis Monotone Selection Theorem is stated as follows (see Topkis[78], Theorem 2.5.2 for a proof):

Proposition 10 (Veinott[81]; Topkis[78]): Suppose $X$ is a nonempty complete lattice, $T$ a poset, $F(x, t): X \times T \rightarrow 2^{X} \backslash \varnothing$ for each $(x, t) \in X \times T$, and assume that the correspondence $F(x, t)$ is isotone in Veinott's strong induced set order on $X \times T$. Let $G(t)$ be the fixed point correspondence of $F(x, t)$ at $t \in T$; then (a) for each $t \in T$, $\sup G(t)$ and $\inf G(t)$ exist; (b) $\sup G(t)$ and $\inf G(t)$ are isotone in $t \in T$; $(c)$ If, in addition, $\sup G(t)<\inf G(t \prime)$ for $t<t$, then $\sup G(t)$ and $\inf G(t)$ are strictly increasing in $t$ on $T$.

Second, consider the case of WCS. There are many alternative sufficient conditions under which fixed point problems exhibit WCS. Different forms of sufficient conditions are provided in Veinott[81] and Smithson[72]. We consider some additional isotone selection theorems that prove useful in the study of WCS in economic applications. These theorems apply in cases where the range of the fixed point correspondence does not necessarily possess the sublattice structure required to apply the Veinott-Topkis monotone selection theorem. For the first proposition, instead of assuming that the correspondence is isotone in Veinott's strong set order jointly in $(x, t)$, we

\footnotetext{
${ }^{6} \mathrm{~A}$ well-known reference for monotone comparative statics in economics is Milgrom and Shannon [53]. However, their results built on prior results in operations research and reported in Veinott [80] and Topkis [76]. See Veinott's [81] lecture notes and Topkis [78].
} 
assume that $F(x, t)$ is ascending in Veinott's weak set relation in $x$ for each $t \in T$. We also assume that the fixed point correspondence has the following structure: (i) $G(t): T \rightarrow 2^{Y} \backslash \varnothing$ is a nonempty and chain subcomplete, and (ii) $G(t)$ is ascending in Veinott's weak set order. We now state Veinott's weak monotone selection theorem:

Proposition 11 (Veinott[81], Theorem 5) Let $X$ be a lattice, $T$ be a partially ordered set. Assume that $G(t): T \rightarrow 2^{X} \backslash \varnothing$ is a chain subcomplete correspondence that is ascending in the Veinott's weak set relation. Then, (a) $G(t)$ admits an isotone selection. If, in addition, we assume $G(t)$ is meet- (respectively, join-) sublattice-valued for each $t \in T$, then (b) the isotone selection is $\wedge G(t)$ (respectively, $\vee G(t)$ ).

Veinott proves more versions of the above isotone selection theorem assuming stronger hypotheses than (a), e.g., $G(t)$ quasi-sublatticed valued for each $t \in T$, but with weaker hypotheses than assumed for result (b). We present two different set of sufficient conditions for the existence of WCS from Smithson[72].

Proposition 12 (Smithson[72], Theorem 1.7 ): Let $X$ be a partially ordered set, $T$ a set, and let $G(t): T \rightarrow 2^{X}$ be a nonempty correspondence that is ascending in Smithson's weak set relation (C1) (respectively, (C2)) in $(x, t)$. If, in addition, $\sup G(t) \in G(t)$ (respectively, $\inf G(t) \in G(t)$ ) for all $t \in T$, the there is an isotone selection, namely $g(t)=\sup G(t)$ (respectively, $g(t)=\inf G(t))$.

We now define Range Majorizing Condition (RMC) and Range Intersection Property (RIP) that are required for stating the second isotone selection theorem. We say a correspondence $F(x)$ satisfies Range Majorizing Condition if for $C=\left\{z \mid x_{1} \leq z \leq x_{2}\right\}, x_{1} \leq x_{2}$, when $F(x) \cap C \neq \varnothing, \sup (F(x) \cap$ $C) \in F(x) \cap C$.Further, if for any $x_{1} \leq x_{2}, y\left(x_{1}\right) \in F\left(x_{1}\right)$ and $y\left(x_{2}\right) \in F\left(x_{2}\right)$ such that $y\left(x_{1}\right) \leq y\left(x_{2}\right)$, and for all $x \in\left[x_{1}, x_{2}\right], F(x) \cap\left[y\left(x_{1}\right), y\left(x_{2}\right)\right] \neq \varnothing$ then we say that $F(x)$ has the Range Intersection Property (RIP).

Proposition 13 (Smithson[72], Theorem 1.9) Let $X$ be a partially ordered set which contains an element $x^{u} \in X$ that is a least upper bound in $X$. If for each $t \in T, G(t): T \rightarrow 2^{Y}$ is nonempty and satisfies conditions MCSC, $R I P$, and $R M C$, then $G(t)$ admits an isotone selection. 
We make a final remark on last two propositions. The proofs of each propositions relies heavily on an application of the Axiom of Choice (namely, the Zorn's lemma). In principle, this can be a serious problem for developing constructive methods that address the question of approximating monotone selections. Recently, alternative methods are developed for the results in Smithson [72] that do not rely upon the Axiom of Choice (see, Jachymski[40], Theorem 2.21). Also, Heikkila and Reffett[37] develop chain methods for computing particular selections that are not based on either the Axiom Schema Replacement or the Axiom of Choice. These extensions are important if one wants to avoid the non-constructive nature of the monotone selection results based on applications of the Axiom of Choice.

\section{An Economy with Classical Technology}

We generalize Brock and Mirman[15] to allow for more general "distorted classical" stochastic technologies. In these economies, time is discrete and indexed by $t \in T=\{0,1,2, \ldots\}$. There is a continuum of ex ante and $e x$ post identical infinitely-lived households. The only form of uninsured risk is aggregate production function shock and production in each state is assumed to be constant returns to scale in private returns. Therefore, the value of all firms is zero in equilibrium. Each period households are endowed with a unit of time which is supplied inelastically in competitive markets. For simplicity, we assume uncertainty comes in the form of a finite state, first-order Markov process denoted by $\theta_{t} \in \Theta$, with stationary transition probabilities $\chi\left(\theta, \theta^{\prime}\right)$. Let the set $\mathbf{K} \subset \mathbf{R}_{+}$contain all feasible values for the aggregate endogenous state variable $K$, i.e., the capital to labor ratio, and define the product space $\mathbf{S}: \mathbf{K} \times \Theta$. Since the household also enters each period with an individual level of the endogenous state variable $k$, the individual capital to labor ratio, we denote the state of a household by the vector $s=(k, S) \in \mathbf{K} \times \mathbf{S}$.

The preferences are represented by a period utility index $u\left(c_{i}\right)$, where $c_{i} \in \mathbf{K} \subset \mathbf{R}_{+}$is period $i$ consumption. Letting $\theta^{i}=\left(\theta_{1}, \ldots, \theta_{i}\right)$ denote the history of the shocks until period $i$, a household's lifetime preference is defined over infinite sequences indexed by date and history $\mathbf{c}=\left(c_{\theta^{i}}\right)$ and is,

$$
U(\mathbf{c})=E_{0}\left\{\sum_{i=0}^{\infty} \beta^{i} u\left(c_{i}\right)\right\},
$$

where $E_{0}$ is the mathematical expectation with respect to the probability structure of the shocks over the infinite horizon. We impose the following assumption on preferences: 
Assumption - P1 : The utility function $u: \mathbf{K} \mapsto \mathbf{R}$ is bounded, twice continuously differentiable, strictly increasing, strictly concave. In addition, marginal utility, $u^{\prime}(c)$ satisfies the standard Inada conditions:

$$
\lim _{c \rightarrow o} u^{\prime}(c)=\infty \text { and } \lim _{c \rightarrow \infty} u^{\prime}(c)=0 .
$$

We assume that the output available to the household in the current period can be represented by the function $F(k, 1, K, 1, \theta, t)=f(k, K, \theta ; t)$, where $t$ is a parameter that is possibly infinite dimensional (e.g., a continuous mapping that represents distortions and thus influences technology). We assume that this production function is evaluated at equilibrium employment levels with $n=N=1$ and make the following assumptions on technology:

Assumption - T1 The production function $F(k, n, K, n, \theta, t)$ is such that:

(i) $F(k, n, K, n, \theta ; t)$ is constant returns to scale in $(k, n)$ for each $(K, N, \theta, t)$ such that $F(0,1, K, 1, \theta, t)=f(0, K, \theta ; t)=0$ for all $K \in \mathbf{K}, \theta \in \not{E}$ and $t \in \mathbb{T}$.

(ii) $f(k, K, \theta, t)$ is twice continuously differentiable, strictly increasing in $(k, K)$ and strictly concave in its first argument.

(iii) $f_{1}(K, K, \theta ; t)$ is weakly decreasing (i.e., non-increasing) in $K$.

(iv) There exist $\hat{k}(\theta)>0$ such that $f(\hat{k}(\theta), K, \theta ; t)+(1-\delta) \hat{k}(\theta)=\hat{k}(\theta)$ and $f(k, K, \theta ; t)<k$ for all $k>\hat{k}(\theta)$ and for all $\theta \in \Theta$.

The restrictions on the primitives in Assumptions P1 and T1 are standard. As we consider some baseline comparative statics issues, we consider the economy studied in Coleman [19]. In this setting, there is a state contingent capital income tax; in addition, we allow for nonconvexities in production in social returns. The distorted reduced-form technology $f$ can be written as follows:

$$
f(k, K, \theta ; t)=\left(1-t_{1}(K, \theta)\right) g(k, K, \theta)+t_{2}(K, \theta),
$$

where $g$ is also a reduced-from distorted classical production function, the parameters $t_{1}(K, \theta): \mathbb{S} \rightarrow[0,1]$ and $t_{2}(K, \theta)$ can be interpreted as the statecontingent tax and a lump sum transfer, respectively. If we define the standard lexicographic order relation on the set of parameter vectors $t \in \mathbb{T}$ as $t^{\prime}(K, \theta) \succeq t(K, \theta)$ if either $t_{1}^{\prime}(K, \theta)>t_{1}(K, \theta)$ for all $S \in(K, \theta) \in \mathbf{K} \times \Theta=\mathbf{S}$, 
or $t_{1}^{\prime}(K, \theta)=t_{1}(K, \theta)$ and $t_{2}^{\prime}(K, \theta) \geq t_{2}(K, \theta)$, then $f(k, K, \theta ; t)$ is increasing in $t$.

We make the following assumption on the nature of distortion:

Assumption - D1: The functions $t_{1}(K, \theta)$ and $t_{2}(K, \theta)$ are Lipschitz continuous on $\mathbf{K} \times \Theta=\mathbf{S}$.

In developing our existence arguments, we fix $t \in \mathbf{T}$. (and, for the moment suppress notation). For any given $t \in \mathbf{T}$, define the household's feasible correspondence to be $\Gamma(k, K, \theta)$ where $\Gamma$ defines the set of actions $\left(c, k^{\prime}\right)$ that satisfy the standard budget constraint:

$$
c+k^{\prime}=f(k, K, \theta) ; \text { and } c, k^{\prime} \geq 0 .
$$

Under Assumption T1, $\Gamma(k, K, \theta)$ is a "well-behaved" nonempty correspondence for each $s=(k, K, \theta) \in \mathbf{K} \times \mathbf{S}$. In particular, as $f$ is continuous and isotone, we conclude that $\Gamma$ is a non-empty, compact and convex-valued, continuous correspondence for each state $s$ that is ascending in $(k, K, \theta)$ for each $t$ in the set inclusion order on $2^{\mathbf{K x K}}$ along an equilibrium restriction where $k=K$ and a balanced budget for the government.

Let $\mathbf{C}(\mathbf{S})$ denote the space of continuous functions $h(S): \mathbf{S} \rightarrow \mathbf{K}$ equipped with the standard uniform norm topology (i.e., $\|h\|=\sup _{S \in \mathbb{S}}|h(S)|$ ) and pointwise Euclidean partial order where $\mathbf{S}$ is a compactum, and let $\mathbf{C}^{+}(\mathbf{S})$ be its cone. To construct the household's decision problem, consider that aggregate capital-labor ratio evolves according to:

$$
K^{\prime}=h(K, \theta) \in \mathbf{C}^{+}(\mathbf{S}), 0 \leq h \leq f,
$$

where for any given $t, h(S): \mathbf{S} \rightarrow \mathbf{K}$ is continuous in both its arguments, increasing in $K$ for each $\theta$. The household solves the following dynamic program:

$$
J(s)=\sup _{\left(c, k^{\prime}\right) \in \Gamma(s ; t)}\left\{u(c)+\beta \int_{\Theta} J\left(s^{\prime}\right) \chi\left(\theta, d \theta^{\prime}\right)\right\} .
$$

Standard arguments prove the existence of a $J \in \mathbb{V}$ that satisfies this functional equation, where $\mathbb{V}$ is a space of bounded, continuous, real valued functions with the sup norm (see, for instance, Stokey, Lucas and Prescott[73]). In addition, under assumptions P1-T1, following the argument in Mirman and Zilcha[56] (lemma 1) $J$ is differentiable in $k$.

We define an recursive equilibrium as follows:

Definition: A (recursive) competitive equilibrium for this economy consists of a parameter vector $\left(t_{1}, t_{2}\right)$, a value function for the household $J(s)$, 
and the associated individual decisions $c$ and $k^{\prime}$ such that: (i) $J(s)$ satisfies the household's Bellman equation (1), and $c, k^{\prime}$ solve the optimization problem in the Bellman's equation given t; (ii) all markets clear: i.e., $k^{\prime}=h(S)=K^{\prime}$ and (iii) the government budget balances.

\subsection{The Existence of MEDPs}

The second welfare theorem does not apply in this economy. Therefore, the social planning approaches to characterizing MEDPs do not suffice. We adopt an alternative strategy, the so-called "Euler equation approach".7 To facilitate our construction, we consider a stronger version of Amann's theorem in Proposition 7, Section 3. This result is proved in Morand and Reffett [57] and considers Amann's theorem for isotone transformations of equicontinuous fixed point spaces.

Proposition 14 Let $E$ be an equicontinuous fixed point space of continuous functions, each defined on a compact set $X$, equipped with the sup continuous uniform topology and the pointwise partial Euclidean order. Let $[\bar{y}, \widehat{y}]$ be a closed suborder interval in E. Suppose that $A:[\bar{y}, \widehat{y}] \rightarrow[\bar{y}, \widehat{y}]$ is an isotone, continuous map. Then $A$ has a maximal fixed point $\widehat{x}$ and $\widehat{x}=\lim _{n \rightarrow \infty} A^{n} \widehat{y}$, and the sequence $\left\{A^{n} \widehat{y}\right\}_{n=0}^{\infty}$ is decreasing.

Proof: See Morand and Reffett[57], Proposition 2.

To construct existence of recursive equilibrium, we define a candidate nonlinear operator $A$ whose fixed points coincide with a MEDP. The Euler equation associated with the optimal policy function in Bellman's equation (1) along an equilibrium trajectory where $k=K$ (appealing to the MirmanZilcha envelope condition) generates the following necessary and sufficient condition for a recursive competitive equilibrium: the existence of a function $c^{*}(K, K, \theta)=c^{*}(K, \theta)$ such that

$u^{\prime}\left(c^{*}(K, \theta)\right)=\beta \int_{\not} u^{\prime}\left[c^{*}\left(F(K, \theta)-c^{*}(K, \theta), \theta^{\prime}\right)\right] r\left(F(K, \theta)-c^{*}(K, \theta), \theta^{\prime}\right) \chi\left(\theta, d \theta^{\prime}\right)$.

\footnotetext{
${ }^{7}$ This is in contrast to the "value function" or the "Bellman equation" approach, in which one looks for a fixed point of the Bellman's operator in the space of value functions. In a non-smooth environment, the Bellman equation approach is useful while the Euler equation approach need not be.
} 
Here, $F(K, \theta)=f(K, K, \theta ; t)$ and $r(K, \theta)=f_{1}(K, K, \theta ; t)$ for notational simplicity.

Definition: $\mathbf{H}^{0}$ is the set of consumption functions $h$ such that:(i). $h: \mathbf{S} \rightarrow \mathbf{K}$;(ii). $\quad 0 \leq h(K, \theta) \leq F(K, \theta)$ for all $(K, \theta) \in \mathbf{S}$;(iii). $0 \leq$ $h\left(K^{\prime}, \theta\right)-h(K, \theta) \leq F\left(K^{\prime}, \theta\right)-F(K, \theta)$ for all $K^{\prime} \geq K,\left(K, K^{\prime}\right) \in \mathbf{K} \times \mathbf{K}$ and all $\theta$.

Equip $\mathbf{H}^{0}$ with the standard sup uniform metric topology; and adopt the Euclidean partial order $\geq$ induced by the cone structure of $\mathbf{C}^{+}(\mathbf{S})$. That is, $h^{\prime} \geq h$ if and only if $h^{\prime}(K, \theta) \geq h(K, \theta)$ for all $(K, \theta) \in \mathbf{S}$. The following lemma summarizes some important properties of the space $\mathbf{H}^{0}$.

Lemma 15 Under assumption T1, (i) $\mathbf{H}^{0}$ is a closed, convex, equicontinuous order interval of continuous function (e.g., a convex compact order interval); (ii) $\mathbf{H}^{0}$ is a complete lattice.

Proof: (i) See Coleman[19] Proposition 3. (ii) See Morand and Reffett[57], Lemma 1.

To construct a recursive equilibrium, we define a nonlinear operator $A h$ based on an equilibrium version of the Euler equation. To do this, consider any $h \in \mathbf{H}^{0}, h>0$, and any $(K, \theta)$ :

Definition: The operator $A h(K, \theta)=\left\{y \mid y\right.$ : for $h>0, u^{\prime}(y)=\beta \int_{\Theta} u^{\prime}(h(F-$ $\left.\left.y, \theta^{\prime}\right), \theta^{\prime}\right) r\left(F-y, \theta^{\prime}\right) \chi\left(\theta, d \theta^{\prime}\right)$; if $h=0$ in any $(K, \theta)$, we set $\left.A h(K, \theta)=0\right\}$.

The following lemma lists a few key properties of the operator $A$ :

Lemma 16 Under Assumptions P1, T1, and D1:(i) For any $h \in \mathbf{H}^{0}$, and any $(k, \theta)$, there exists a unique $A h(k, \theta) ;(i i) \quad A$ maps $\mathbf{H}^{0}$ into itself (e.g., is a transformation of $\left.\mathbf{H}^{0}\right)$;(iii) $A$ is continuous on $\mathbf{H}^{0}$; (iv) there exists a maximal fixed point $h^{*} \in \mathbf{H}^{0}$ and the sequence $\left\{A^{n} F\right\}$ converges uniformly to $h^{*}$; and, (v) the maximal fixed point is strictly positive.

Proof: The proofs of (i)-(iii) are in Coleman[19] (Proposition 4). Claim (iv) follows directly from Proposition 14. Claim (v) follows from a standard dynamic programming argument that is presented in the main theorem in Greenwood and Huffman[34] p 615

It is important to note that neither (i), (ii), nor (iii), rely on compactness of the state-space, and are therefore valid under Assumptions P1 and T1 only. We can now state our existence result for MEDPs. 
Proposition 17 Under Assumptions P1, T1 and D1, there exists a recursive equilibrium.

Proof: Follows from Lemma 15 and Lemma 16.

\subsection{The Uniqueness of MEDPs}

We next consider the uniqueness of MEDPs. We present a new result that dispenses with the $k_{0}$-monotonicity condition used commonly in the literature and obtain uniqueness from the concavity of the fixed point operator. Let $C^{+}$be a cone in a real Banach space $C$, and consider a transformation $A: C^{+} \rightarrow C^{+}$. We say an operator $A: C^{+} \rightarrow C^{+}$is $e$-concave if there exists non-zero $e \in C^{+}$, such that (i) for an arbitrary non-zero $c \in C^{+}$the inequalities $\alpha e \leq A c \leq \beta e$, where $\alpha$ and $\beta$ are positive, are valid and (ii) for every $c \in C^{+}$such that $\alpha_{1}(c) e \leq c \leq \beta_{1}(c) e$ with $\left(\alpha_{1}(c), \beta_{1}(c)\right) \gg 0$, and there is a number $\eta(c, t)>0$ such that $A(t c) \geq(1+\eta) t A c$ for any $t \in(0,1)$. An operator is said to be pseudo-concave on $C^{+}$if for all $t \in(0,1), c \in$ $C^{+}, c>0, A t c>>t A c$. Let $C^{+}$be a solid cone, the operator $A: C^{+} \rightarrow C^{+}$ is strongly sublinear if Atc $>>t A c$ for all non-zero $c \in C^{+}$and $0<t<1$. (See Guo and Lakshmikantham[35], Definition 2.2.2).

Let $H \subset \mathbf{C}^{+}(\mathbf{S})$ be an compact order interval where $\mathbf{C}^{+}(\mathbf{S})$ is the space of positive continuous functions on the compact set $\mathbf{S}=\mathbf{K} \times \Theta$. We say an operator $A$ is $k_{0}$-monotone on $H$ if it is (i) isotone on $H$, and (ii) if for any strictly positive fixed point $h_{1}$, there exists a $k_{0}>0,0 \leq k_{1} \leq k_{0}$ and $h_{2} \in H$ such that $h_{2} \leq h_{1}$, for all $k \geq k_{1}$, and $h_{1}(k, \theta) \geq A h_{2}(k, \theta)$ all $k \geq k_{1}$, for all $\theta$. To construct new sufficient conditions for uniqueness, we first construct the operator $\widehat{A}$ as in Coleman[21] but we prove additional properties of this operator that are useful for our argument that are not in Coleman. We define the set of functions $\mathbf{M}$ as follows:

Definition: $\mathbf{M}=\left\{m: \mathbf{R}_{+} \times \Theta \rightarrow \mathbb{R} \mid(\mathrm{i}) \quad m\right.$ is continuous, (ii) for all $(K, \theta) \in \mathbf{R}_{+} \times \Theta, 0 \leq m(K, \theta) \leq F(K, \theta)$ and (iii) for any $K=0$, $m(K, \theta)=0\}$

Endow $\mathbf{M}$ with the standard partial pointwise order and the $C^{0}$ uniform topology. We note that $\mathbf{H}^{0}$ and $\mathbf{M}$ can be directly related to each other by a simple mapping. For $m \in \mathbf{M}$, consider the function $\Psi(m(K, \theta))$ implicitly defined by,

$$
u^{\prime}[\Psi(m(K, \theta))]=\frac{1}{m(K, \theta)}, \text { for } m>0,0 \text { elsewhere. }
$$


Clearly, $\Psi$ is continuous, increasing, $\lim _{m \rightarrow 0} \Psi(m)=0$, and $\lim _{m \rightarrow F(K, \theta)} \Psi(m)=$ $F(K, \theta)$.Using the function $\Psi$, for any $m>0$, we denote the solution (for $y$ ) to the following equation by $\widehat{A} m(K, \theta)$,

$$
\widehat{Z}(m, y, K, \theta)=\frac{1}{y}-\beta E_{\theta}\left[\frac{H\left(F(K, \theta)-\Psi(y), \theta^{\prime}\right)}{m\left(F(K, \theta)-\Psi(y), \theta^{\prime}\right)}\right]=0,
$$

and set $\widehat{A} m=0$ when $m=0$. Since $\widehat{Z}(m, y, K, \theta)$ is strictly decreasing and continuous in $y$ and $\lim _{y \rightarrow 0} \widehat{Z}(m, y, K, \theta)=\infty$ and $\lim _{y \rightarrow F(K, \theta)} \widehat{Z}(m, y, K, \theta)=$ $-\infty$, for each $m(K, \theta)>0$, with $K>0$, and $\theta \in \not{\text { }}$, there exists a unique $\widehat{A} m(K, \theta)$.

It is easy to show that to each fixed point of the operator $A$ corresponds a fixed point of the operator $\widehat{A}$. Indeed, consider $x$ such that $A x=x$ and define $y=\frac{1}{u^{\prime}(x)}$ (or, equivalently $\Psi(y)=x$ ). It is also easy to verify that $A m \subset \mathbf{M}$ and is monotone on $\mathbf{M}$. By definition, for all $(K, \theta), x$ satisfies,

$u^{\prime}(x(K, \theta))=\beta E_{\theta}\left\{H\left(F(K, \theta)-x(K, \theta), \theta^{\prime}\right) \times u^{\prime}\left(x\left(F(K, \theta)-x(K, \theta), \theta^{\prime}\right)\right)\right\}$.

Substituting the definition of $y$ into this expression yields

$$
\frac{1}{y}=\beta E_{\theta}\left\{\frac{H\left(F(K, \theta)-\Psi(y(K, \theta)), \theta^{\prime}\right)}{y\left(F(K, \theta)-\Psi\left(y(K, \theta), \theta^{\prime}\right)\right)}\right\},
$$

which shows that $y$ is a fixed point of $\widehat{A}$.

We are now prepared to prove our new uniqueness result:

Proposition 18 Under Assumptions P1, T1, D1, (i) The operator $\widehat{A}$ is strongly sublinear; (ii) $\hat{A}$ has at most one strictly positive fixed point; and, (iii) there exists a unique recursive equilibrium in $\mathbf{H}^{0}$.

Proof: (i). First note both $\mathbf{H}^{0}$ and $\mathbf{M}$ are order intervals in solid cones of continuous functions defined on a compact set. Therefore since $\widehat{Z}$ is strictly decreasing in its second argument, a sufficient condition for strong sublinearity of $\hat{A} m$ is:

$$
\widehat{Z}(t m, t \widehat{A} m, K, \theta)>\widehat{Z}(t m, \widehat{A} t m, K, \theta)=0 .
$$

By definition,

$$
\widehat{Z}(t m, t \widehat{A} m, K, \theta)=\frac{1}{t \widehat{A} m}-\beta E_{\theta}\left\{\frac{H\left(F(K, \theta)-\Psi(t \widehat{A} m(K, \theta)), \theta^{\prime}\right)}{\operatorname{tm}\left(F(K, \theta)-\Psi(t \widehat{A} m(K, \theta)), \theta^{\prime}\right)}\right\}
$$


so that,

$$
t \widehat{Z}(t m, t \widehat{A} m, K, \theta)=\frac{1}{\widehat{A} m}-\beta E_{\theta}\left\{\frac{H\left(F(K, \theta)-\Psi(t \widehat{A} m(K, \theta)), \theta^{\prime}\right)}{m\left(F(K, \theta)-\Psi(t \widehat{A} m(K, \theta)), \theta^{\prime}\right)}\right\} .
$$

Since $\Psi$ is increasing and $H\left(K^{\prime}, \theta^{\prime}\right) / m\left(K^{\prime}, \theta^{\prime}\right)$ is decreasing in $K^{\prime}$,

$$
\begin{gathered}
\frac{1}{\widehat{A} m}-\beta E_{\theta}\left\{\frac{H\left(F(K, \theta)-\Psi(t \widehat{A} m(K, \theta)), \theta^{\prime}\right)}{m\left(F(K, \theta)-\Psi(t \widehat{A} m(K, \theta)), \theta^{\prime}\right)}\right\} \\
>\frac{1}{\widehat{A} m}-\beta E_{\theta}\left\{\frac{H\left(F(K, \theta)-\Psi(\widehat{A} m(K, \theta)), \theta^{\prime}\right)}{m\left(F(K, \theta)-\Psi(\widehat{A} m(K, \theta)), \theta^{\prime}\right)}\right\}=0,
\end{gathered}
$$

and $\widehat{Z}(t m, t \widehat{A} m, K, \theta)>0$ so it must be the case that $\hat{A} t m>t \hat{A} m$. Therefore, $\hat{A} m$ is strongly sublinear.

(ii) As $\hat{A} m$ is strongly sublinear, by theorem 2.2.1(a) in Guo and Lakshmikantham[35], we conclude $\hat{A} m$ is $e$-concave. As $\hat{A} m$ is additionally increasing on $m$, by Guo and Lakshmikantham[35] (Theorem 2.2.2) we conclude $\hat{A} m$ has at most a single strictly positive fixed point in M. By the lemma 16(iv), $A h$ has a strictly positive fixed point $\mathbf{M}$. Therefore, we conclude by the definition of $\hat{A} m$, that $\hat{A} m$ has a unique strictly positive fixed point in $\mathbf{M}$.

(iii) As the $\hat{A} m$ has a unique strictly positive fixed point in $M$, by the definition of $\hat{A} m$ and the fact that $\hat{A}[M]$ is isomorphic to $A\left[\mathbb{H}^{0}\right]$, we conclude there is a strictly positive fixed point $h^{*} \in \mathbf{H}^{0}$. By an standard argument (e.g., see Vailakis[79], Section 5), interiority of consumption and investment (along with the fact $h^{*} \in \mathbf{H}^{0}$ ) is sufficient in this case to support prices in $l_{+}^{1} \backslash\{0\}$

Remark 19 A careful reading of our new proof of uniqueness in Proposition 18 provides a new method for characterizing the existence of recursive equilibrium with prices in $l_{+}^{1} \backslash\{0\}$. This proof differs substantially from the proof of sufficient conditions for interiority often used in the existing literature for the existence of strictly positive fixed point (e.g., the proof given in Greenwood and Huffman[34], that is used also in Coleman[20] and Datta, Mirman and Reffett[22] for models with elastic labor supply). The equivalence of strictly positive fixed points can be shown. Note that, as $\mathbf{M}$ is an order interval in a normal cone of continuous functions, $\hat{A} m$ is e-concave and isotone. Then by a theorem in Guo and Lakshmikantham[35] (Theorem 2.2.3), we conclude $\hat{A} m$ is a cone compression. ${ }^{8}$ And, by a remark in Guo

\footnotetext{
${ }^{8}$ See the discussion in Guo and Lakshmikantham ([35], p64-5) for a discussion of cone compressions.
} 
and Lakshmikantham[35] (p65), we arrive at the complete characterization of the existence of a strictly positive fixed point: $\hat{A} m$ is a cone compression if and only if $\hat{A} m$ has a strictly positive fixed point.

\subsection{Monotone Comparison Theorems using Euler Equation Methods}

In this section, we construct monotone comparison theorems using Euler equation methods. The monotonicity of the mapping $A$ in lemma 16 can be exploited to derive strong comparative statics (SCS) results on the space of deep parameters $t \in T$ using the selection theorems in Section 3. The set of equilibrium is a non-empty complete lattice, so, in the absence of the uniqueness result, comparative statics analysis requires defining orders on both the set of parameters and on the set of equilibrium. We show that the set of equilibrium is ascending in the strong set order of Veinott in $t$, consequently, we conclude that the minimal and maximal fixed points are also monotonic in $t$.

Proposition 20 Suppose that the assumptions of lemma 16 and Proposition 17 are satisfied for each mapping $A_{t}$ belonging to the set $\left\{A_{t}: \mathbf{H}^{0} \rightarrow \mathbf{H}^{0}\right.$, $t \in T\}$, where $\left(T, \geq_{T}\right)$ is a poset, and $G(t)$ is the fixed point correspondence of $A_{t}$. If $A_{t}$ is isotone in $t$, that is if $t^{\prime} \geq_{T} t$ implies that, for all $x$ in $X$, $A_{t^{\prime}} x \geq A_{t} x$, then $G(t)$ is ascending in Veinott's strong set order $\geq_{s}$ on $2^{\mathbf{H}^{0}}$ and the minimal and maximal fixed points (respectively, $\wedge G(t)$ and $\vee G(t)$ ) of $A_{t}$ are isotone mappings into $\mathbf{H}^{0}$ on $T$.

Proof: The claims follow from the proof in Morand and Reffett[57], Theorem 2, noting that $G(t)$ is isotone in Veinott's strong set order, a direct implication of Proposition 10.

For an application of this result, consider a perturbation in the discount rate $\beta$. Since the right side of the Euler equation in (2) is increasing in $\beta$, as a consequence, the root $y^{*}(K, \theta, h, t)=A_{t=\beta}(c)$ that defined the operator, is increasing in $\beta \in]-1,0\left[=T\right.$, where $T$ is endowed with the dual order $\geq_{T}$ on the real line (i.e., $\beta^{\prime} \geq_{T} \beta$ if $\beta^{\prime} \leq \beta$ ). By Proposition 20, the maximal and minimal fixed points increase with $t$ (i.e., decrease with $\beta$ ). By Proposition 18 , the set of MEDPs increase in the pointwise strong set order $\geq_{s s}$ and there is a unique isotone selection. 
For another application, consider the tax rate $t \in T$, where $T$ is the set of continuous functions $t(K, z) \in[0,1]$ that are monotone in $K$. Endow $T$ with the standard pointwise Euclidean order for a space of functions, i.e., $t^{\prime} \geq_{T} t$ if $t^{\prime}(K, z) \geq t(K, z)$ for all $(K, z)$. Then $A_{t^{\prime}} c \geq A_{t} c$ in the order defined on $E$ and the equilibrium set (the set of fixed points of the operator $A_{t}$ ) is isotone in $t$ the strong set order. Again, by Proposition 18, we can obtain a unique isotone selection on $T$ from the set of MEDPs.

\section{An Economy with Nonclassical Technology}

We now allow for more general versions of bounded nonconvex production technologies, linear preferences, Markov technology shocks and a role for public policy. By "distorted nonclassical" production technologies, we mean two cases: the reduced-form production function $f(k, K, \theta)$ is such that (i) $f_{1}(k, K, \theta)$ is not decreasing in $k$ when $k=K$, and/or (ii) $f$ is not necessarily constant returns to scale in private inputs. In (ii), that there is an issue with interpreting exit and entry conditions in the industry within the equilibrium model but we ignore the industry dynamics. Uncertainty (and much of the model) is as before. Preferences and technologies are denoted as before, except we now have weaker assumptions:

Assumption - P2: The utility index $u(c) \in \mathbf{U}$ where $\mathbf{U}$ consists of all $u(c): \mathbf{K} \mapsto R$ that are bounded, continuous, strictly increasing, and either strictly concave on $\mathbf{K}$ or linear on $\mathbf{K}$.

Assumption - T2: The aggregate production functions $f \in F$, where $F$ consists of isotone functions $f(k, K, \theta): \mathbf{K} \times \mathbf{K} \times \mathbf{\Theta}$, each space ordered with pointwise Euclidean orders, $f$ is continuous in $k$ and there exists $\hat{k}(\theta)>$ 0 such that $f(\hat{k}(\theta), \theta)+(1-\beta) \hat{k}(\theta)=\hat{k}(\theta)$ and $f(k, \theta)<k$ for all $k>\hat{k}(\theta)$ for all $\theta \in \boldsymbol{\Theta}$; and $f$ is twice differentiable its arguments. ${ }^{9}$

We impose a joint restriction on the curvature of $u(c)$ relative to the complementarity of the equilibrium distortion in $f(k, K, \theta)$. This restriction is used only for our methods when $f \in F$ such that $f(K, K, \theta)$ is not concave in $K$. (See section 6.2 for further discussion of this point, and how this restriction can be eliminated in the case $f(K, K, \theta)$ is concave.)

\footnotetext{
${ }^{9}$ We also refer to an isotone function as a monotone function.
} 
Assumption - PT1: The utility index $u \in U$ and the aggregate production technology $f \in F$ are such that $u^{\prime}(\gamma(K)) f_{1}(k, K, \theta)$ is isotone in $K$ for each function $\gamma(K)$ where $\gamma(K)$ satisfies $0 \leq \gamma\left(K^{\prime}\right)-\gamma(K) \leq$ $f\left(k, K^{\prime}, \theta\right)-f(k, K, \theta)$ for $K^{\prime} \geq K .^{10}$

We need a regularity property on the stochastic process of shocks.

Assumption - M1: The transition matrix $\chi \in \Xi$ is an irreducible Markov process that satisfies the standard Feller property.

When discussing the long-run properties of a Markovian equilibrium (and equilibrium comparative statics on limiting distributions), it is useful to restrict attention to a subset of economies where we can prove Markovian dynamics are jointly monotone in $(K, \theta)$. Therefore, we note the following additional assumptions:

Assumption - PT2: The class $U$ and $F$ have $u^{\prime}(\gamma(\theta)) f_{1}(k, K, \theta)$ are isotone in $\theta$ for each $\gamma(\theta)$ such that $0 \leq \gamma\left(\theta^{\prime}\right)-\gamma(\theta) \leq f\left(k, K, \theta^{\prime}\right)-$ $f(k, K, \theta) .{ }^{11}$

Assumption - M2: The measure $\chi \in \Xi$ is stochastically increasing (or equivalently, totally positive of order 2). ${ }^{12}$

The case of optimal growth under uncertainty in embedded in above assumptions. Our results are more general than those obtained for the optimal growth model with Markov shocks in Hopenhayn and Prescott[39]. Although they claim a more general result, a careful reading of their proofs reveals that Hopenhayn and Prescott can only claim sufficient conditions for monotone controls in the optimal growth model with Markov shocks when production functions are the fixed-coefficient, Leontief-type. ${ }^{13}$ Note that,

\footnotetext{
${ }^{10}$ If one is willing to adopt the slightly stronger complementarity condition related to the one mentioned in Hopenhayn and Prescott [39] (i.e., $u^{\prime \prime}(c) f_{1} f_{2}+u^{\prime}(c) f_{12} \geq 0$ ), we can allow $u(c)$ in assumption $\mathrm{P} 2$ to be concave (but not necessarily linear).

${ }^{11}$ This assumption includes the case for Markov shocks mentioned (but not studied) in Hopenhayn and Prescott [39] for the optimal growth model.

${ }^{12}$ See Topkis [78] for a definition of stochastically increasing.

${ }^{13}$ This key problem with applying a key theorem of Topkis [78] (Theorem 2.7.6) also arises in Amir [5]. In this paper, if one follows his proofs, one realizes that the author can only claim the existence of monotone controls in the nonclassical optimal multisector growth model when production functions are either (i) Leontief or (ii) defined on domains where the inputs are chained. Our approach using generalized envelopes can be easily applied in the multisector growth model to obtain more general sufficient conditions for monotone controls in multisector models than found in Amir's work.
} 
we can dispense with assumption M1 or M2 for the optimal growth case. Also, if the class of shocks $\chi \in \Xi$ consists of a collection of independent and identically distributed random variables, then we obtain joint monotonicity for decentralized Markovian equilibrium under weaker conditions. We can completely dispense with Assumption PT2, and we still obtain joint monotonicity of the decentralized MEDPs. For the optimal growth case, we only require $u \in U$ concave, and $f(K, \theta)$ monotone in $(K, \theta)$.

\subsection{The Parameter Space and Household Decision Problems}

Consider the existence of MEDPs under the assumptions P2, T2, PT1 and M1. We begin by defining the fixed point space we use to compute MEDPs.

Definition: $\mathbf{C}_{1}=\left\{h \mid 0 \leq h(K, \theta) \leq f(K, K, \theta) \forall(K, \theta) ; h\left(K^{\prime}, \theta\right)-\right.$ $h(K, \theta) \geq 0$ if $K^{\prime} \geq K, h$ measurable on $\left.\mathbf{S}\right\}$.

Here $h \in \mathbf{C}_{1} \subset \mathbf{B}(\mathbf{S}), \mathbf{S}$ is a compact partially ordered topological space with the pointwise Euclidean order (and the usual topology on $\mathbf{R}^{n}$ ). $\mathbf{B}(\mathbf{S}$ ) is the set of bounded functions $\mathbf{S}^{\mathbf{K}}$ endowed with the standard pointwise Euclidean order and $C^{0}$ uniform topology, and $\mathbf{C}_{1}$ consists of all positive functions that are isotone in $K$, measurable, and socially feasible, monotone in $K$.

Assume that households take as given the recursion $h$ on per-capita aggregate capital stock $K$, which is used to compute future returns on capital (and, therefore, factor prices),

$$
K^{\prime}=h(K, \theta) \in \mathbf{C}_{1}, 0 \leq h \leq f .
$$

If we make additionally assume PT2 and M2, we obtain stronger characterizations of Markovian equilibrium. For that situation, consider the space,

Definition: $\mathbf{C}_{2}=\left\{h \mid h(K, \theta) \in \mathbf{C}_{1}\right.$ that are jointly isotone in $\left.(K, \theta)\right\}$.

Clearly $\mathbf{C}_{2}(\mathbf{S})$ is a closed sublattice of $\mathbf{C}_{1} \subset \mathbf{B}(\mathbf{S})$. The spaces $\mathbf{C}_{1}$ and $\mathbf{C}_{2}$ are used to find Markovian equilibrium for economies without and with assumptions PT2 and M2, respectively. We next prove a lemma that is useful in constructing a Markovian equilibrium.

Lemma 21 Both $\mathbf{C}_{1}$ and $\mathbf{C}_{2}$ are convex and subcomplete in $\mathbf{B}(\mathbf{S})$. 
Proof: See Mirman, Morand, and Reffett[54], lemma 1.

Therefore, $\mathbf{C}_{1}$ (respectively, $\mathbf{C}_{2}$ ) is a natural place to pose the existence question.

We now characterize the best response mapping of households facing an aggregate environment $h \in \mathbf{C}_{1}$, under the assumptions P2, T2, PT1 and M1. Consider a household entering the period in state $p=\left(p_{c}, \theta\right) \in$ $P=\mathbf{K} \times \mathbf{K} \times \boldsymbol{\Theta}, p_{c}=(k, K) \in \mathbf{K} \times \mathbf{K}$, facing an aggregate economy with aggregate dynamics (and prices) summarized by the function $h \in \mathbf{C}_{1}$. Let consumption and investment be given as $a=(c, y) \in A \subset \mathbf{K} \times \mathbf{K}$. The value function for the household is a function $v^{*}(p, h)$ that is a solution of the functional equation:

$$
v^{*}(p ; h)=\sup _{a \in \Gamma(p)}\left\{u(c)+\beta \int_{\Theta} v^{*}\left(y, h(K, \theta), \theta^{\prime} ; h\right) \chi\left(\theta, d \theta^{\prime}\right)\right\},
$$

where the feasible correspondence $\Gamma(p)=\{a \mid c+y \leq f(p), c, y \geq 0\}$. In order to study the existence of a $v^{*}$ that satisfies the above functional equation, consider the operator $B^{C}$ :

$$
\begin{aligned}
B^{C} v(p ; h)= & \sup _{a \in \Gamma(p)}\{u(c)+ \\
& \left.\beta \int_{\Theta} v\left(y, h(K, \theta), \theta^{\prime} ; h\right) \chi\left(\theta, d \theta^{\prime}\right)\right\} .
\end{aligned}
$$

Here the operator $B^{C}$ is defined on the space $V_{c}=\left\{v(p ; h): \mathbf{P} \times \mathbf{C}_{1} \rightarrow \mathbf{R}\right.$, $v$ bounded in $(k, K, \theta, h)$, isotone in $p$ for each $h$, continuous in $k$ for each $(K, \theta, h)\}$. Equip $V_{c}$ with the standard $C^{0}$ topology (and the associated uniform metric) and the pointwise Euclidean partial order. $V_{c}$ is a complete metric space. Lemma 22 provides a set of results characterizing the unique function $v^{*}$ that satisfies (4):

Lemma 22 Under assumptions P2, T2, PT1 and M1, (i) $B^{C} v \subset V_{c}$; (ii) there exists a unique $v^{*} \in V_{c}$ that satisfies the Bellman equation (4); and, (iii) the fixed point $v^{*}$ is strictly increasing in $p$ for each $h \in \mathbf{C}_{1}$.

Proof: A standard argument. See Stokey, Lucas, and Prescott[73].

We now use lattice programming to further characterize the value function. ${ }^{14}$ Define the optimal solution associated with $v^{*}(p ; h)$ by $a^{*}(p, h)$,

\footnotetext{
${ }^{14}$ We assume familiarity in this section with the basic terminology of lattice programming (supermodular functions etc.). Important references are LiCalzi and Veinott [49], Veinott [81], and Topkis [78].
} 


$$
\begin{aligned}
a^{*}(p ; h)= & \left\{\arg \sup _{c, y \in \Gamma(p)}\{u(c)+\right. \\
& \left.\left.\beta \int_{\Theta} v\left(y, h(K, \theta), \theta^{\prime} ; h\right) \chi\left(\theta, d \theta^{\prime}\right)\right\}\right\} .
\end{aligned}
$$

To characterize the optimal solution $a^{*}(p, h) \subset 2^{A}$, we define a set of partial orders over choices of consumption $c$ and investment $y$. The class of partial orders is referred to as "direct value" orders and was pioneered in the work of Antoniadou[7]. To fix ideas, consider the simple two good version of the consumer decision problem. Assume that the relative price is one. Define a collection of direct value orders for unit price for $a=(c, y) \in A \subset \mathbf{K} \times \mathbf{K}$ (denoted by $\geq_{v i}$, where $i \in I$, an index set) as follows: $a, a^{\prime} \in A$, we say $a^{\prime} \geq_{v i} a$ if and only if $c^{\prime}+y^{\prime} \geq_{e} c+y$ and $a^{\prime} \geq_{L i} a$. Here $\geq_{e}$ is referred to as the value quasi-order on $A$, and $\geq_{L i}$ is the standard lexicographic order defined using the index set $I=\{c, y\}$ on $A \subseteq R_{+}^{2}$. We use this collection of valuation lattices $\left(A, \geq_{v i}\right)$ to model the action space for the stochastic growth model $A \subseteq R_{+}^{2}$. When indexing the lexicographic order in the valuation order by $c$, we refer to the resulting lattice, on the commodity space $\left(A, \geq_{v c}\right)$, as the consumption value lattice. We also make reference to the investment value lattice when indexing the lexicographic order in the valuation order by investment $\left(A, \geq_{v y}\right)$. Antoniadou [7] shows that the space $\left(A, \geq_{v i}\right)$ is (i) a partially ordered set for each $i \in I=\{c, y\}$, and (ii) $\geq_{v i}$ induces a lattice structure on $A$ for each $i=c, y$. Define, $\Gamma(p)=\{a \mid c+y \leq m, c, y \geq$ $0, m=f(p)\} \subseteq A$ when $\left(A, \geq_{v i}\right) i=1,2$.Under assumptions P2, T2, PT1 and M1, and each index $i=c, y$, the feasible correspondence $\Gamma(p)$ is (i) an isotone mapping $P \rightarrow 2^{A}$ in the strong set order $\geq_{a}$ endowed with either of the partial orders $i=c, y$; and (ii) it is a nonempty, continuous, compact, convex, and complete sublattice for each $p \in P$.

We turn next to a characterization of supermodular functions on the collection $\left(A, \geq_{v i}\right)$. In the next lemma, we characterize additively separable supermodular objectives on the direct value lattices $\left(A, \geq_{v i}\right)$. Let $U(x, y)$ : $A \rightarrow R$ on the lattice $\left(A, \geq_{v i}\right)$.

Lemma 23 Assume $U(x, y)=u_{1}(x)+u_{2}(y)$, where each $u_{i}($.$) is isotone for$ $i=1,2$. Then $(i) U(x, y)$ is supermodular (strictly supermodular) on the $x$ valuation lattice $\left(A, \geq_{v x}\right)$ if and only if $u_{2}(y)$ concave (strictly concave), (ii) $U(x, y)$ is supermodular (strictly supermodular) on the collection $\left(A, \geq_{v I}\right)$ for $I=x, y$ if and only if both $u_{1}(x)$ and $u_{2}(y)$ are concave (strictly concave). 
Proof: See Mirman, Morand, and Reffett[54] Lemma 4.

Now, we consider sufficient conditions for monotone controls $a^{*}(p, h)$ from (5) to be isotone in the Euclidean order on $A$. The parameters of interest are $p_{c}=(k, K)$ and $h \in \mathbf{C}_{1}$. A major obstacle to studying the dynamic complementaries in (5) is characterizing sufficient conditions for preserving supermodularity under maximization. One set of sufficient conditions for preserving supermodularity under maximization on arbitrary projections to the parameter space is found in Topkis[78] (Theorem 2.7.6). This set of sufficient conditions cannot be applied in growth models with multidimensional parameter spaces as they require the graph of the feasible correspondence to be sublattice valued in the powersets of $A \times P$; a condition not available in growth models unless the production function is Leontief. We, therefore, do not follow this line of argument. We develop results on generalized envelope conditions found in the literature on nonsmooth analysis. See Clarke[17] (chapter 2) and Rockafellar and Wets[66]. This approach is used in Askri and LeVan[8] who study envelope theorems in the multisector optimal growth model with nonclassical technologies. Unfortunately, however, their results only apply to economies for which the optimal solutions are strictly interior. In our framework, their methods cannot be directly applied. We extend Askri and LeVan[8] results to economies without boundary restrictions, such as Inada conditions. Our method is based on Gauvin and Dubeau[33].

Let $p \in P$. Note that $P$ is a convex sublattice. Consider the subspace of value functions $V(p) \subset V_{c}$ consisting of the $v(k, K, \theta, h) \in V_{c}$ with the following additional restrictions:

(i) $v(p)$ is supermodular in $p_{c}=(k, K) \in P_{c}$ for each $\theta$;

(ii) Lipschitz in $k$ with the Lipschitz constant,

$$
L=\sup _{c, k, K, \theta, h} \mid\left\{u^{\prime}(c) f_{1}(k, K, \theta), u^{\prime}(0) f_{1}(k, K, \theta)+\varepsilon \mid,\right.
$$

where $\varepsilon=\beta \int u^{\prime}\left(f\left(k, h(K, \theta), \theta^{\prime}\right)\right) f_{1}\left(k, h(K, \theta), \theta^{\prime}\right) \chi\left(\theta, d \theta^{\prime}\right)-u^{\prime}(0)$. The subset $V$ is a closed subset of the complete metric space of functions $V_{c}$.Also, recall that supermodularity is closed under pointwise limits (see Topkis[78], lemma 2.6.1). We have the following monotonicity result,

Proposition 24 Let us assume P2, T2, PT1 and M1 and let $v \in V(p)$. Then (i) the optimal solution $a^{*}(h ; p): \mathbf{C}_{1} \rightarrow 2^{A}$ is ascending in $h$ in the strong set order $\geq_{a}$ on the investment valuation lattice $\left(A, \geq_{v y}\right)$; and, (ii) the maximal and minimal selections for investment $a_{y}^{u}(h ; p)=\max _{y} a^{*}(h ; p)$ and $a_{y}^{l}=\min _{y} a^{*}(h ; p)$ are measurable isotone functions from $\mathbf{C}_{1} \rightarrow A$. 
Proof: See Mirman, Morand, and Reffett[54], Theorem 5.

Notice that monotonicity on the investment lattice $\left(A . \geq_{v y}\right)$ implies that investment monotonicity on the Euclidean lattice $\left(A, \geq_{E}\right)$. Proposition 24 implies that the extremal selections of the best response map are monotone on the space $\mathbf{C}_{1}$ for each $(k, K, \theta)$.Corollary 25 shows that the extremal selections form self maps to the space $\mathbf{C}_{1}$ :

Corollary 25 Assume P2, T2, PT1 and M1, let $v^{*} \in V$ in equation (4) and for each $\theta \in \Theta$; then for $h \in \mathbf{C}_{1}$ (i) the optimal solution $a^{*}\left(p_{c}, \theta ; h\right)$ is ascending from $P_{c} \rightarrow 2^{A}$ in the strong set order $\geq_{a}$ on the investment valuation lattice $\left(A, \geq_{v y}\right)$; and, (ii) the minimal and maximal selections for investment $a_{y}^{u}\left(p_{c}, \theta ; h\right)=\max _{y} a^{*}\left(p_{c}, \theta ; h\right)$ and $a_{y}^{l}=\min _{y} a^{*}\left(p_{c}, \theta ; h\right)$ are measurable isotone functions from $P_{c} \rightarrow$ A. Under additional assumptions PT2 and M2, and for $h \in \mathbf{C}_{2}$, (iii) the optimal solution $a^{*}(p ; h)$ is ascending from $P$ to $2^{A}$ in the strong set order $\geq_{a}$ on the investment valuation lattice $\left(A, \geq_{v y}\right)$; and, (iv) the minimal and maximal functions for investment $a_{y}^{u}(p)=\max _{y} a^{*}(p)$ and $a_{y}^{l}=\min _{y} a^{*}(p)$ are measurable isotone functions from $P \rightarrow A$.

In the proof of Proposition 24 and Corollary 25 in Mirman, Morand, and Reffett[54], they also prove a new envelope theorem that generalizes the result in Mirman and Zilcha [56], Amir, Mirman, and Perkins[6], and Askri and LeVan[8]. With this envelope, it is straightforward to check that the right side of (4) at a solution $v^{*}$ has all the requisite complementary structure to obtain isotone increasing controls in Veinott's strong set order $\geq_{s}$ (namely, the requisite increasing differences between the controls and the parameters). Given that this new generalized envelope is of independent interest, we present the argument for its existence.

We need to define some terms. A correspondence $\Gamma(p)$ is said to uniformly compact near $p$ if there is a neighborhood $N(p)$ of $p$ such that the closure of $\cup_{p^{\prime} \in N(p)} \Gamma\left(p^{\prime}\right)$ is compact. Given the continuity of $f$ in $p$ for economies $\Delta \in E$, one can prove that the feasible correspondence on (4), $\Gamma(p)$, is uniformly compact near $p$. Rewrite the constraints in (4), more generally, as $\Gamma(p)=\{a \mid g(a, p) \leq 0\}$ where $g(a, p)$ is the set of implicit constraints defined in (4). We say a pair $(a, p) \in \operatorname{gr} \Gamma(p)$ satisfies the Mangasarian-Fromowitz regularity conditions (or, are MF-regular) if there exists a direction $r \in R^{2}$ such that the Jacobian $\nabla_{a} g(a, p) r<0, g(a, p)=$ $0 .{ }^{15}$ Here $\operatorname{gr} \Gamma(p)$ is the graph of $\Gamma(p)$. In our problem, the constraints are

\footnotetext{
${ }^{15}$ As all constraints are inequalities, we are writing that MF regularity constraint qualifi-
} 
additively separable with constant gradients in the controls, for any pair $(a, p) \in A \times P$; therefore each point $(a, p)$ is $M F$ regular. Therefore, any optimal solution $\left(a^{*}(p, h), p\right) \in g r \Gamma(p)$ is a MF-regular point. Further, because these coefficients do not change as a function of $a$, we also note that we have a stronger constraint qualification present, namely that the basis elements $\nabla_{a} g\left(a^{*}(p, h), p\right)$ are linearly independent. Therefore, our problem also satisfies the so-called "linear independence" (LI) constraint qualification discussed in Gauvin and Dubeau[33].

Next, note a few properties of Bellman operator $B^{c}$. Let $v \in V$. We know that the feasible correspondence $\Gamma(p): P \rightarrow 2^{A}$ is a continuous, strong set order ascending correspondence in $p=(k, K, z)$ for each $h \in \mathbf{C}_{1}$. Further, for each $p, \Gamma(p)$ is nonempty, compact, convex and subcomplete in $\left(A, \geq_{v i}\right)$ for $i=c, y$. As $u(c)$ is Lipschitz (as its $C^{1}$ with bounded gradient on any neighborhood of $\mathbf{K}$ that is strictly interior), and the sum of two Lipschitz functions is Lipschitz, we conclude that the objective is Lipschitz in $(c, y)$ for each $(p, h)$. By a standard application of Berge's maximum theorem[11] p.116), the value function $B^{C} v$ is continuous in $k$, and the optimal solutions $a^{*}(p, h)$ form a nonempty, compact-valued correspondence for each $(p, h)$. Noting the continuity of the objective, $a^{*}$ is also upper hemi-continuous correspondence in $k$. As the order on $P$ pointwise Euclidean, when $P$ is endowed with the standard metric/topology, $P$ is a Banach lattice with a continuous lattice structure. Also note that $\left(A, \geq_{v i}\right) i=c, y, A$ has a continuous lattice structure, and $A=\mathbf{K} \times \mathbf{K}$ is Hausdorff. Therefore, by Debreu[26], the optimal solutions $a^{*}(p): P \rightarrow \rightarrow 2^{A}$ is are upper-measurable. (See also Hopenhayn and Prescott[39] for discussion of upper-measurability).

We next prove that the value function is locally Lipschitz. This result is needed to obtain a global Lipschitz estimate that is useful for proving $B^{c} v \in V$.

Proposition 26 The Bellman operator, $B^{c}: P \times \mathbf{C}_{1} \rightarrow R$, is locally Lipschitz near $k>0$, for each $(K, z, h)$ and $v^{*}(k, K, \theta, h)$ is Clarke differentiable in its first argument for each $(K, \theta, h)$.

Proof: We have two cases.

Case 1: The optimal solutions $a^{*}(p, h)$ are strictly interior in $A=\mathbf{K} \times \mathbf{K}$; i.e., for all $a(p, h) \in a^{*}(p, h), a(p, h) \in \operatorname{int}\left(R_{+}^{2}\right)$

cations for a problem with only inequality constraints, i.e, we do not require for all binding constrants, say $h(a, p)=0$, to satisfy that the direction $r$ is othrogonal to $\nabla_{a} h(a, p)$ where $h$ is the collection of all the equality constraints. 
By a result in Amir, Mirman, and Perkins[6] (lemma 3.3) left and right Dini derivatives exist in $k$ for each $(K, z, h)$ and are bounded. By Rockafellar[65] (Proposition 5), $B^{c} v$ is therefore locally Lipschitz with a upper estimate of the Lipschitz modulus of $L_{v}(p, h)=\sup _{p, k>0}\left\{B^{c+} v, B^{c-} v\right\} \leq L$ where for example $B^{c+}$ is the right Dini at $(p, h), k>0$.

Case 2: The optimal solutions $a^{*}(p, h)$ is such that there is an $a(p, h) \in$ $a^{*}(p, h)$ not interior.

Using a standard Lagrangian approach, the operator $B^{c} v$ is given as follows: for $h \in \mathbf{C}_{1}$,

$$
\begin{aligned}
B^{c} v & =\sup _{a, \lambda, \varphi_{c}, \varphi_{y}} L(a, p, h) \\
& =\sup _{a, \lambda, \varphi_{c}, \varphi_{y}} u(c)+\beta \int v\left(y, h(K, \theta), \theta^{\prime}\right) \chi\left(\theta, d \theta^{\prime}\right)+\lambda(f-c-y)+\varphi_{c} c+\varphi_{y} y
\end{aligned}
$$

where $\lambda, \varphi_{c}, \varphi_{y}$ are the multipliers associated with the respective constraints that define $\Gamma(p)=\{a \mid c+y \leq f(k, K, z), c \geq 0, y \geq 0\}$. As (i) each element of $\left(a^{*}(p, h), p\right)$ is MF-regular such that it also satisfies the condition (LI) and (ii) the primitive data of the problem is Lipschitz, by corollary 4.4 in Gauvin and Dubeau[33], $B^{c} v$ has bounded right and left Dini derivatives in $k$ with $B^{c+} v_{k}(k, K, z, h)=\max _{a \in a^{*}(p)} \nabla_{k}^{+} L(a, p, h) \leq L$, and $B^{c-} v_{k}(k, K, z, h)=\max _{a \in a^{*}(p)} \nabla_{k}^{-} L(a, p, h) \leq L$ for $k>0, p \in P$. Then by Gauvin and Dubeau[33] (Theorem 5.1), $B^{c} v$ is locally Lipschitz in $k>0$, $p \in P, h \in \mathbf{C}_{1}$ (see also Rockafellar[65], Proposition 5)

This generalized Clarke envelope is a critical step: the economies that satisfy assumptions $\mathrm{P} 2, \mathrm{~T} 2, \mathrm{PT} 1$ and $\mathrm{M} 1$, the value function $v^{*}(k, K, \theta, h)$ has increasing differences in $(k ; K, h)$ for each $\theta$. If, in addition, we assume $\mathrm{PT} 2$ and M2, then we obtain $v^{*}$ also having increasing differences in $(k ; \theta)$.

\subsection{The Existence of MEDPs}

We prove the existence of a complete lattice of Markovian equilibrium. Noting the dependence of best responses on the environment (in the next section we conduct monotone comparative statics on the space of environments), we denote a correspondence,

$$
T h(K, \theta)=\left\{a(K, K, \theta ; h) \mid a \text { any monotone selection in } a^{*} \text { in }(6)\right\}
$$

We state some useful properties of the correspondence $T h$. In particular, we focus on the sublattice structure of its range: 
Lemma 27 Under assumptions P2, T2, PT1 and M1, Th $\subset \mathbf{C}_{1}$, Th is ascending on $\mathbf{C}_{1}$ in the strong set order $\geq_{a}$ to $2^{C_{1}}$ and is complete-latticed valued; with additional assumptions PT2 and M2, Th: $\mathbf{C}_{2} \rightarrow 2^{\mathbf{C}_{2}}$ is ascending in the strong set order $\geq_{a}$ and $T$ is complete lattice valued.

Recalling the Veinott-Zhou version of Tarski's theorem in Proposition 2 , we obtain our first result (the proof follows directly from Lemma 27 and Proposition 2),

Proposition 28 Under the assumptions P2, T2, PT1 and M1, the set of fixed points $\varphi_{T}^{*}$ is a nonempty complete lattice in $\mathbf{C}_{1}$; with additional conditions PT2 and M2, the set of fixed points $\varphi_{T}^{*}$ is a nonempty complete lattice in $\mathbf{C}_{2}$.

\subsection{Monotone Comparison Theorems via Lattice Program- ming Methods}

We first point out straightforward monotone comparison results with respect to changes in the discount rate and shock process. Consider ordered perturbations of the discount rate $\beta$ and/or uncertainty $\chi \in \Xi$ (where the ordered perturbation of measure $\chi$ take place in a setting of first order stochastic dominance). Using variations of existing arguments (e.g., Amir, Mirman and Perkins[6] (Theorem 5.1) and Hopenhayn and Prescott[39] (corollary 7) for perturbations in $\beta$ and $\chi$, respectively), we obtain a Veinott strong set order monotone comparative statics result in the pointwise Euclidean order from the extremal selections of agent investment decisions for investment $a_{y}^{*}(p, h ; \beta, \chi)$, under assumptions $\mathrm{P} 2, \mathrm{~T} 2, \mathrm{PT} 1$ and M1. Then by the Veinott-Topkis SCS theorem, we obtain Veinott strong-set order fixed point correspondence comparison with the operator $T h$ by $\varphi_{T}^{*}(\beta, \chi)$ and have the SCS via Proposition 10, Section 3. We conclude that the fixed point correspondence $\varphi_{T}^{*}(\beta, \chi)$ exhibits strong set order comparative statics, i.e., $\varphi_{T}^{*}:(0,1) \times \Xi \rightarrow 2^{\mathbf{C}_{1}}$ is a strong set order increasing correspondence.

To study monotone comparative statics with respect to the space of reduced-form distorted production functions, our argument requires the development of a set of partial orders that is suitable for ordering the envelope conditions for agents' decisions. This partial order involves "gradient monotonicity" conditions. Infinite dimensional single crossing properties relative to a space of payoff functions for a collection of parameterized dynamic programs have been studied by Lovejoy[50]. Consider the order on the space 
of technologies $F: f^{\prime} \geq_{F} f$ when $u\left(f^{\prime}(k, K, z)\right)-u(f(k, K, z))$ is increasing in $k$, for each $(K, z)$ with $f^{\prime}-f=0$, when $k=0,(k, K, z) \in P$, and $P$ is compact. ${ }^{16}$ Observe the following: (a) $\left(F, \geq_{F}\right)$ is a partially ordered space antisymmetry follows given $f$ vanishes at zero), (b) $f^{\prime} \geq_{F} f$ implies $f^{\prime}(p) \geq f(p)$ for all $p$ in the pointwise Euclidean order, and, (c) $f^{\prime} \geq_{F} f$ implies that the gradients, $\partial_{k} f^{\prime}(p) \geq \partial_{k} f(p)$, are pointwise ordered in the Euclidean order.

Proposition 29 provides some monotone comparative statics results. We have examples of SCS and WCS. As a prerequisite to stating this result, we define a few terms that are useful in characterizing the order theoretic properties of the random dynamical systems. Let $\mathbf{M}(\mathbf{K} \times \mathbf{Z})$ be the space of finite measures on $\mathbf{K} \times \mathbf{Z}$, endow $\mathbf{M}$ with the stochastic dominance partial order, that is $\lambda^{\prime} \geq_{M} \lambda$ if for every monotone, measurable, nonnegative, and bounded function $f: \mathbf{K} \times \mathbf{Z} \rightarrow R_{+}, \int f \lambda^{\prime}(d k \times d \gamma) \geq \int f \lambda(d k \times d \gamma)$. Hopenhayn and Prescott[39] (Proposition 3) show that when this order is restricted to the space of monotone, measurable, bounded, and nonnegative functions, $\left(\mathbf{M}, \geq_{M}\right)$ is a partially order set under the stochastic dominance order $\geq_{M}$. When viewed from a topological perspective, Dudley[29] (Proposition 11.3.2) provides a metric under which $\mathbf{M}$ is a compact metric space. Let $(\mathbf{K} \times \mathbf{Z}, \mathcal{B}(\mathbf{K}) \times \mathcal{B}(\mathbf{Z}))$ be measurable spaces where $\mathcal{B}($.$) denotes the Borel$ measurable subsets. Consider the adjoint operator $J(\lambda ; h): \mathbf{M}(\mathbf{K} \times \mathbf{Z}) \times \mathbf{C}_{2}$ $\rightarrow \mathbf{M}(\mathbf{K} \times \mathbf{Z})$ defined as,

$$
J(\lambda ; h)(A \times B)=\int I_{A}(h(k, z)) \chi(z, B) \lambda(d k \times d z),
$$

where $I_{A}$ is the indicator function for a measurable set $A \in \mathcal{B}(\mathbf{K}), B \in \mathcal{B}(\mathbf{Z})$. For each $h \in \mathbf{C}_{2}$, define the fixed point correspondence for the operator $J$ $(\lambda ; h)$ to be $\Psi_{J}^{*}(h)=\{\lambda \in \mathbf{M}, \lambda=J(\lambda, h)\}$. Define $\lambda_{m}(h)=\min \Psi_{J}^{*}(h)$, and let $\varphi_{J}^{*}(f)$ be the set of invariant distributions associated with the set of Markovian equilibrium $\varphi_{T}^{*}(f)$, for any production function $f \in F$.We have,

Proposition 29 Assume P2, T2, PT1 and M1, let $f \in\left(F, \geq_{F}\right)$. Then (i) the correspondence of Markovian equilibrium, $\varphi_{T}^{*}(f): F \rightarrow 2^{C_{1}}$ is ascending in the strong set order $\geq_{a}$. Further, with additional assumptions PT2 and M2, (ii) the set of equilibrium invariant distributions $\varphi_{J}^{*}(f): F \rightarrow 2^{M}$ is

\footnotetext{
${ }^{16}$ Note that the partial order defined with respect this difference is increasing in each component of $p$. We fix $(K, z)$, and emphasize the role of $k$ in our discussion below.

Also, similar orders can be developed to obtain monotone controls in consumption, relative to the space of production function by developing the obvious dual argument using the dual order relative to capital.
} 
ascending in the Smithson-weak set relation $\geq_{\text {as }}$ and admits a monotone selection on F; and (iii) the dynamics exhibit monotone comparative dynamics in the Smithson-weak set relation.

Note that standard arguments can be used to prove the existence of an invariant distribution for a Markovian equilibrium in $\varphi_{T}^{*}(f)$. The main contribution of Proposition 29 concerns comparative dynamics results on the space of equilibrium correspondence. The problem of ruling out limiting distributions that do not have ergodic sets on a strictly positive support is nontrivial. We leave further characterization of a stationary Markovian equilibrium for future work. Note that, isotone selections in $\varphi_{J}^{*}(f)$ exist as one can easily check the conditions of Smithson's weak isotone selection theorem discussed in Section 3, Proposition 12.

\section{An Economy with Elastic Labor Supply}

We revisit the model with classical technology (Section 4) and allow for elastic labor supply. This model is formulated as in Datta, Mirman and Reffett[22]. As in the previous sections, we consider a continuum of household/firms populating the economy. Uncertainty and market structure are also similar to that in Sections 4 and 5 but the household cares about leisure. For each period and state, preferences are represented by a period utility in$\operatorname{dex} u\left(c_{i}, l_{i}\right),\left(c_{i}, l_{i}\right) \in R_{+} \times[0,1]$. Letting $\theta^{i}=\left(\theta_{1}, \ldots, \theta_{i}\right)$ denote the history of the shocks until period $i$, the households lifetime preferences are additively separable and defined over infinite sequences indexed by dates and histories,

$$
U(\mathbf{c}, \mathbf{l})=E_{0}\left\{\sum_{i=0}^{\infty} \beta^{i} u\left(c_{i}, l_{i}\right)\right\} .
$$

Here $E_{0}$ is the expectation with respect to the probability structure of future histories of the shocks $\theta^{i}$ given the transition matrix $\chi$. The period utility function $u: R \times[0,1] \mapsto R$, satisfies,

Assumption - P3: The period utility index $u(c, l)$ is such that:

(i) $u(c, l)$ is continuously differentiable, strictly increasing, and strictly concave in $(c, l)$.

(ii) The partial derivatives $u_{c}(c, l)$ and $u_{l}(c, l)$ satisfy the Inada conditions:

$$
\lim _{c \rightarrow 0} u_{c}(c, l)=\infty, \lim _{c \rightarrow \infty} u_{c}(c, l)=0, \lim _{l \rightarrow 0} u_{l}(c, l)=\infty .
$$

(iii) The second partials satisfy, 


$$
\frac{u_{c c}}{u_{c}} \leq \frac{u_{l c}}{u_{l}}, \frac{u_{l l}}{u_{l}} \leq \frac{u_{c l}}{u_{c}} .
$$

The assumptions on period utility are standard. (See Datta et al[22] and Vailakis[79] for discussion of this assumption). Note that condition P3(iii) can be thought of as "normality". It also means that the marginal rate of substitution $\frac{u_{l}}{u_{c}}$ is non-decreasing in $c$ and $\frac{u_{l}}{u_{c}}$ is non-increasing in $l$. And this is slightly stronger than quasi-concavity of the period utility function (we assume it to be strictly concave) because it implies,

$$
u_{c}^{2} u_{l l}+u_{l}^{2} u_{c c} \leq 2 u_{c} u_{l} u_{c l},
$$

which is a necessary condition for quasi-concavity. This condition is automatically satisfied if $u_{c c}(c, l)<0, u_{l l}(c, l)<0$ and $u_{c l}(c, l) \geq 0$. If the cross-partial is negative, the condition restricts its magnitude.

Each household is endowed with a unit of time, and enters into a period with an individual stock of capital $k$. We assume a decentralization where firms do not face dynamic decision problems. Households own the firms as well as both the factors of production, and they rent these factors of production in competitive markets. In addition, to allow for externalities in the production process, as in previous sections, we assume that the production technologies of the firms to depend on per capita aggregates. Assume that technology satisfies,

Assumption - T3: The production function $f: \mathbf{K} \times[0,1] \times \mathbf{K} \times[0,1] \times$ $\Theta \rightarrow R$ satisfies,

(i) $f(0,0, K, N, \theta)=0$ for all $(K, N, \theta) \in \mathbf{K} \times[0,1] \times \Theta$,

(ii) $f(k, n, K, N, \theta)$ is continuous, increasing, differentiable; in addition, it is concave and homogeneous of degree one in $(k, n)$.

(iii) $f(k, n, K, N, \theta)$ also satisfies the standard Inada conditions in $(k, n)$ for all $(K, N, \theta) \in \mathbf{K} \times[0,1] \times \Theta$; i.e.,

$$
\begin{aligned}
& \lim _{k \rightarrow 0} f_{k}(k, n, K, N, \theta)=\infty, \\
& \lim _{n \rightarrow 0} f_{n}(k, n, K, N, \theta)=\infty, \\
& \lim _{k \rightarrow \infty} f_{k}(k, n, K, N, \theta)=0 .
\end{aligned}
$$

(iv) There exists a $\hat{k}(\theta)>0$, such that $f(\hat{k}(\theta), 1, \hat{k}(\theta), 1, \theta)+(1-\beta) \hat{k}(\theta)=$ $\hat{k}(\theta)$ and $f(k, 1, k, 1, \theta)<k$ for all $k>\hat{k}(\theta)$, for all $\theta \in \Theta$.

Assumption T3 is standard in the stochastic growth literature (see Brock and Mirman[15]). With the initial stock $k_{0}$, we can define $\bar{k}=\max \left\{k_{0}, \sup _{\theta} \hat{k}(\theta)\right\}$ 
and the state space for the capital stock and output can be defined on the compact set $\mathbf{K} \subseteq[0, \bar{k}]$. Let $\mathbf{K}_{+}$denote the set of strictly positive values for $k$.

\subsection{The Household Decision and Equilibrium}

Imagine a consumer faced with a choice problem of a single good and leisure in the first stage. The objective is to maximize the difference between the level of utility and the expenditure to obtain that level of utility (see Topkis[78]). Normalizing on the price of consumption goods, consumers take the price of leisure $w(K, \theta)$, the level of per capita consumption $C$, and the per capita leisure level $L(C, K, \theta)$, as given. Here $C \in \mathbf{K}, w: \mathbf{K} \rightarrow R_{++}, L$ $: \mathbf{K} \times \mathbf{S} \rightarrow[0,1]$, and $L$ is a continuously once-differentiable function, and as in previous sections, $\mathbf{S}:=\mathbf{K} \times \Theta$. Given $w$, the household solves,

$$
v(C, K, L, \theta))=\sup _{l \in[0,1]} \frac{u(C, l)}{u_{c}(C, L)}-w l,
$$

for each $(C, K, L, \theta) \in \mathbf{K}^{2} \times[0,1] \times \not \subset$. Given the assumption P3, standard arguments using the Theorem of the Maximum, establish that the value function $v$ is well-defined and continuous (e.g., see Berge[11], p.115). Further, by the strict concavity of period utility in P3, the optimal policy correspondence associated with $v$ is a singleton. The necessary condition for this first-stage maximization problem is,

$$
\frac{u_{l}\left(C, l^{*}(C, K, \theta)\right)}{u_{c}(C, L)}=w(K, \theta) .
$$

To finish our description of the first stage, we need to determine equilibrium factor prices as functions of the aggregate state variable. We do this from the representative firm's static production problem. Assume that firms maximize profits under perfect competition, i.e., the firms maximize profits subject to given factor prices, say $\bar{r}(K, \theta)$ and $\bar{w}(K, \theta)$, the rental rate for capital and the wage rate, respectively. The factor prices are continuous functions of the aggregate state variable. The representative firm's maximum profit is,

$$
\Pi(\bar{r}, \bar{w}, K, N, \theta)=\sup _{k, n} f(k, n, K, N, \theta)-\bar{r} k-\bar{w} n
$$

where anticipating the standard definition of competitive equilibrium, we set $k=K$ and $n=N(S)$, for $S \in \mathbf{S}$. 
In the second-stage, the household solves a dynamic capital accumulation problem. To describe this problem, we parameterize the aggregate economy facing a typical decision maker. Define to be the space of bounded, continuous functions with domain $\mathbf{S}$ and range $\mathbf{R}_{+}$. To parameterize the household's decision problem, we first describe the aggregate economy.

If the aggregate per capita capital stock is $K$, then households assume a continuous function for per capita labor supply $0 \leq N(S) \leq 1$, and a recursion of the capital stock $K^{\prime}$ is given by,

$$
K^{\prime}=h(S) ; h \in \mathbf{C}^{+}(\mathbf{S}), 0 \leq h \leq f(K, 1-N(S), \theta)
$$

where $\mathbf{C}^{+}(\mathbf{S})$ is as before the space of positive continuous functions on $\mathbf{S}$ with the uniform topology. Using the solution to the household's first stage decision problem (and, imposing equilibrium on the labor market), define the per capita aggregate labor supply $N(S)=1-l^{*}(C, K, \theta)$. Then the aggregate economy consists of functions $\Omega=(w, r, h, C, N)$ from a space of functions with suitable restrictions needed to parameterize the household's decision problem in the second-stage. Assume that the policy-induced equilibrium distortions have the following standard form,

$$
r=\left[1-\pi_{k}(S)\right] \bar{r}, w=\left[1-\pi_{n}(S)\right] \bar{w},
$$

where $\pi=\left[\pi_{k}, \pi_{n}\right]$ is a continuous mapping $\mathbf{S} \rightarrow[0,1) \times[0,1)$. We assume regularity conditions on the distorted prices,

Assumption - D2 :The vector of distortions $\pi=\left[\pi_{k}, \pi_{n}\right]$ is such that the distorted wage $w=\left(1-\pi_{n}(K, \theta)\right) \bar{w}$ and the distorted rental rate $r=\left(1-\pi_{n}(K, \theta)\right) \bar{r}$ satisfy,

(i) $w: \mathbf{K} \times \Theta \rightarrow \mathbf{R}_{+}$is continuous, at least once-differentiable and (weakly) increasing in $K$,

(ii) $r: \mathbf{K}_{+} \times \Theta \rightarrow \mathbf{R}_{+}$is continuous and decreasing in $K$ such that,

$$
\lim _{K \rightarrow 0} r(K, \theta) \rightarrow \infty .
$$

In other words, we assume that the distorted wage and rental rates behave as the non-distorted rates $\bar{w}, \bar{r}$ or the marginal products of labor and capital, respectively. Assumptions D2(i) and P3(iii) imply that leisure increases with higher consumption and decreases with larger capital accumulation.

Next define the lump-sum transfer to each agent, $d(S)=\pi_{k} K+\pi_{n} N(K, \theta)$. Then household's total income is $y(s)=r k+w N+\Pi+d(s)$ where $s$ is the individual household's state, $s=(k, S)=(k, K, \theta)$ and $\Pi$ is profit. Note 
that under assumptions P3, T3 and D2, $y(s)$ is a continuous function. We next define the household's feasible correspondence, $\Psi(s)$, which consists of the set $\left(c, k^{\prime}\right) \in \mathbf{R}_{+}^{2}$ that satisfy,

$$
c+w l^{*}(C, K, \theta)+k^{\prime}=y,
$$

given $(k, K, \theta) \gg 0$. Notice that $\Psi(s)$ is well behaved. In particular since $\Pi$ is continuous, $\Psi$ is a non-empty, compact and convex-valued, continuous correspondence.

Next, we state the second stage decision problem for the household. At the beginning of any period the aggregate state for the economy is given by $S \in \mathbf{S}$. Each household enters the period with their individual capital stock $k \in \mathbf{K}$, so their individual state is $s \in \mathbf{K} \times \mathbf{S}$. Then the households dynamic decision problem is summarized by the Bellman equation,

$$
v(s)=\sup _{\left(c, k^{\prime}\right) \in \Psi(s)} u\left(c, l^{*}(C, K, \theta)\right)+\beta \int_{\Theta} v\left(s^{\prime}\right) \chi\left(\theta, d \theta^{\prime}\right)
$$

Standard arguments show the existence a $v \in \mathbb{V}$ that satisfies this functional equation, where $\mathbb{V}$ is again the space of bounded, continuous functions with the uniform norm. In addition, since $u$ is strictly concave in $c$, standard arguments also establish that $v$ is strictly concave in its first argument, $k$. Once again, from Mirman and Zilcha[56], the strict concavity of $v$ also implies that the envelope theorem applies and the solution $v$ to the Bellman equation is once differentiable in $k$.

We are now prepared to define equilibrium.

Definition: A (recursive) competitive equilibrium for this economy consists of sequences functions $r, w, d$, and $\kappa$; a value function for the household $v(s) \in \mathbb{V}$ and the associated individual decisions $c^{*}(s)$ and $n^{*}(s)$ such that (i) given $r, w, d$ and $\kappa, v(s)$ satisfies the household's Bellman equation (12); (ii) $c^{*}(s)$ solves the right-hand side optimization in the Bellman's equation, $l^{*}(s)=1-n^{*}(s)$ solves the first-stage utility maximization; (iii) all markets clear: i.e., $k^{\prime}=h(S)=K^{\prime}, n^{*}(s)=N(S), c^{*}(s)=C(S)$ and the government budget constraint holds, i.e., $d=\pi_{k} k+\pi_{n} n^{*}$

\subsection{The Existence of Equilibrium}

Before we state the existence problem, we define a number of functions. In equilibrium, $c(s)=C(S), k=K, n=N(S)$, then $y(s)=F(K, \theta)=$ $f\left(K, 1-l^{*}(C(S), K, \theta), \theta\right)+(1-\beta) K$. The next period capital stock, in 
equilibrium, is given as $K^{\prime}=y-C$. Also, for later reference, define $\hat{l}(S)$ as the solution to,

$$
\frac{u_{l}(f(K, 1-\hat{l}(S), \theta), \hat{l}(S))}{u_{c}(f(K, 1-\hat{l}(S), \theta), \hat{l}(S))}=\left(1-\pi_{n}(S)\right) f_{n}(K, 1-\hat{l}(S), \theta) .
$$

Notice that $\hat{l}$ is the amount of leisure that is compatible with no household investment in the first-stage utility maximization. At any (aggregate) state $S$, the maximum possible amount of consumption occurs if $c=f$ and, i.e., if there is no investment. In general, the amount of consumption is less than $f$ and leisure, which is positively related to consumption, is therefore less than $\hat{l}(S)$. That is, for a given state $S, 1-\hat{l}(S)$ is the lower bound for the amount of labor supplied. In addition, $\hat{l}(S)$ is differentiable with respect to $K$, by the implicit function theorem, since the marginal utilities, technology and the distorted wage is differentiable in $K$. Moreover, for the special case, $u_{c l} \geq 0, \hat{l}(S)$ is increasing in $K . \hat{l}(S)$ is also increasing in $K$, for the case $u_{c l}<0$, if

$$
u_{l l}-f_{n} u_{c l}<0, u_{c l}-f_{n} u_{c c}>0 .
$$

The Euler equation, associated with the right side of the Bellman equation (8) above, can be rewritten as,

$$
u_{c}\left(c, l^{*}(c, K, \theta)\right)=\beta \int_{\Theta} u_{c}\left(c\left(K^{\prime}, \theta^{\prime}\right), l^{*}\left(c^{\prime}, K^{\prime}, \theta^{\prime}\right)\right) r\left(K^{\prime}, \theta^{\prime}\right) \chi\left(\theta, d \theta^{\prime}\right) .
$$

Here the ' notation refers to next period value of the particular variable. Given a candidate function $c(S)$, we rewrite the Euler equation (10) in equilibrium as,

$$
\begin{aligned}
u_{c}\left(c, l^{*}(c, K, \theta)\right)= & \beta \int_{\Theta} u_{c}\left(c\left(F_{c}-c, \theta^{\prime}\right), l^{*}\left(c\left(F_{c}-c, \theta^{\prime}\right), K^{\prime}, \theta^{\prime}\right)\right) . \\
& r\left(F_{c}-c, \theta^{\prime}\right) \chi\left(\theta, d \theta^{\prime}\right)
\end{aligned}
$$

where $F_{c}=f\left(K, 1-l^{*}(c(K, \theta), K, \theta), \theta\right)+(1-\beta) K$. We can use equation (11) to define a nonlinear operator that yields a strictly positive fixed point in the space of consumption functions. This fixed point is an equilibrium for the economy.

Define $F^{u}(S)=F^{u}(K, \theta)=f(K, 1-\hat{l}(K, \theta), \theta)+(1-\beta) K$ and consider the following space of functions,

Definition: $\mathbf{H}_{l}=\left\{h: \mathbf{S} \rightarrow \mathbf{K}, h\right.$ continuous, $h(S) \in\left[0, F^{u}(S)\right]$ and $h$ such that $u_{c}\left(h(S), l^{*}(h(S), S)\right)$ is decreasing in $h, u_{c}\left(h(S), l^{*}(h(S), S)\right)$ is decreasing in $K$. 
Equip $\mathbf{H}_{l}$ with the sup norm. Note that the assumption the marginal utility of consumption is decreasing in $h$ means that the space $\mathbf{H}_{l}$ differs from the space of consumption functions studied in Coleman[20]. It is easily verified that for the preferences considered in that paper, the restriction $u_{c}$ decreasing in $h$ is implied. However, since the class of preferences studied in this paper is larger than that studied in Coleman, additional restriction is necessary on the space of consumption functions.

Define the extended real valued mapping $Z: \mathbf{H}_{l} \times \mathbf{Y} \times \mathbf{K} \times \mathbf{Z} \rightarrow \overline{\mathbf{R}}$ where $\mathbf{Y} \subset \mathbf{R}_{+}$, as

$$
\begin{aligned}
Z(h, \zeta, K, \theta) & =\Psi_{1}(\zeta, K, \theta)-\Psi_{2}(h, \zeta, K, \theta) \\
\Psi_{1} & =u_{c}\left(\zeta, l^{*}(\zeta, K, \theta)\right) \\
\Psi_{2}=\beta \int_{\Theta} u_{c}\left(h\left(F_{\zeta}-\zeta, \theta^{\prime}\right), l^{*}\right. & \left.\left(h\left(F_{\zeta}-\zeta, \theta^{\prime}\right), F_{\zeta}-\zeta, \theta^{\prime}\right)\right) r\left(F_{\zeta}-\zeta, \theta^{\prime}\right) \chi\left(\theta, d \theta^{\prime}\right) .
\end{aligned}
$$

Here $F_{\zeta}=f\left(K, 1-l^{*}(\zeta, K, \theta)+(1-\delta) K\right.$. Then define the nonlinear operator $A: \mathbf{H}_{l} \rightarrow \mathbf{H}^{\prime}$ as follows:

$$
A h(K, \theta)=\{\zeta \text { such that } Z(h, \zeta, K, \theta)=0, h>0 ; A h(K, \theta)=0 \text { elsewhere }\}
$$

where $\mathbf{H}^{\prime}$ at this point is an appropriate Banach space.

We discuss some properties of the operator $A$,as defined by equations (12) - (15).

Proposition 30 Under Assumptions P3, T3 and D2, for any $h \in \mathbf{H}_{l}$, there exists a unique $A h=\tilde{h}$ such that $Z(h, \tilde{h}, K, \theta)=0$, for any $(K, \theta)$.

Proof: Datta, Mirman and Reffett[22], Proposition 1.

Proposition 30 implies that for all states, the operator $A h$ is well defined and under the continuity assumptions on preferences, technologies, and distorted prices, continuity of $A h$ is obvious. To study the fixed points of $A$, we first establish that $A$ is a transformation of $\mathbf{H}_{l}$ : i.e., $A: \mathbf{H}_{l} \rightarrow \mathbf{H}_{l}$. It will be convenient to assume

Assumption - P4: The cross-partial of the utility function is nonnegative, that is, $u_{c l} \geq 0$.

Greenwood and Huffman [34] only consider the case where $u_{c l}=0$. Coleman [20] allows for $u_{c l} \geq 0$ and also some cases where $u_{c l}<0$. However, 
he considers a restricted homothetic class of preferences and, in addition, imposes more restrictions (jointly on utility, production functions and distortions) to study the case of negative cross partials of $u$. The same case of negative cross-partials of $u$ can be handled in our setting also. At this stage, we are unable to capture more general cases of negative cross partials of $u$ than Coleman [20], therefore, we focus only on the $u_{c l} \geq 0$ case. And, we have the following:

Proposition 31 Under assumptions P3, P4, T3 and D2, Ah $\subset \mathbf{H}_{l}$.

\section{Proof: Datta, Mirman and Reffett[22], Theorem 1.}

Notice that $\mathbf{H}_{l}$ is a non-empty, convex subset of a space of continuous, bounded real-valued functions but it not equicontinuous, and is therefore not relatively compact. ${ }^{17}$ Since it is well known that the space of all continuous functions on a compactum $X$, denoted by $\mathbf{C}(\mathbf{S})$, with the sup-norm metric is a Banach lattice, $\mathbf{H}_{l}$ is a sublattice in $\mathbf{C}(\mathbf{S})$. Now, a closed subset of continuous, bounded real-valued functions (on a compact domain) equipped with sup-norm metric is compact if and only if it is equicontinuous. The theorem of Arzela and Ascoli (see Dieudonne [27] , p.136-137) says that a set of equicontinuous, pointwise compact subset of the continuous functions is relatively compact.

Define the following subset of $\mathbf{H}_{l}$,

Definition: $\overline{\mathbf{H}}=\left\{h \in \mathbf{H}_{l}\right.$ such that $0 \leq\left|h\left(K_{2}, \theta\right)-h\left(K_{1}, \theta\right) \quad\right| \leq \mid$ $F\left(K_{2}, l^{*}\left(h\left(K_{2}, \theta\right), K_{2}, \theta\right)-F\left(K_{1}, l^{*}\left(h\left(K_{1}, \theta\right), K_{1}, \theta\right) \mid\right.\right.$, for all $\left.K_{2} \geq K_{1} \cdot\right\}$

A standard argument shows that the space of consumption functions $\overline{\mathbf{H}} \subset \mathbf{H}_{l}$ is a closed, pointwise compact, and equicontinuous set of functions. Then by a standard application of Arzela-Ascoli, $\overline{\mathbf{H}}$ is a compact, convex, order interval in $\mathbf{H}_{l}$. Notice that the restriction on consumption in the space $\overline{\mathbf{H}}$ that distinguishes it from $\mathbf{H}_{l}$ implies that the investment function $K^{\prime}=$ $F_{h}-h$ is an increasing functions of the current capital stock $K$ which follows because $F_{h}$ is increasing in $K$ (since $l^{*}$ is decreasing in $K$, the marginal products of capital and labor are positive).

We note some important properties of the operator $A$ and the space $\overline{\mathbf{H}}$,

\footnotetext{
${ }^{17} \mathrm{~A}$ set is relatively compact if its closure is compact.
} 
Proposition 32 Under assumptions P3, P4, T3 and D2, $\overline{\mathbf{H}}$ is a complete lattice and $A$ is a transformation on $\overline{\mathbf{H}}$, i.e., $A h \subset \overline{\mathbf{H}}$.

Proof: See Datta, Mirman, and Reffett[22] Lemma 1 and Theorem 2.

To apply a lattice-theoretic fixed point theorem, we need to verify isotonicity,

Proposition 33 Under assumption P3, P4, T3 and D2, A is isotone on $\mathbf{H}_{l}$.

Proof: Datta et al[22] Theorem 3.

We now restrict the mapping $A$ to the subspace $\overline{\mathbf{H}}$ (which is well-defined since $A$ is continuous, $\overline{\mathbf{H}}$ is compact, order subinterval in $\mathbf{H}_{l}$ and apply a version of Amann's theorem,

Proposition 34 Under assumptions P3, P4, T3 and D2, the set of fixed points of $A: \overline{\mathbf{H}} \rightarrow \overline{\mathbf{H}}$ has a maximal fixed point $A h^{*} \in \overline{\mathbf{H}}$ such that $\lim _{n \rightarrow \infty} A^{n} F \rightarrow A h^{*}=h^{*}$, uniformly..

Proof: Apply Proposition 14; see also Datta et al[22] Proposition 2.

\subsection{The Uniqueness of Equilibrium}

Lastly, we show uniqueness of equilibrium with strictly positive consumption. The proof of strict positivity exploits the strong sublinearity of the operator $A h$ (or, the related operator $\hat{A} m$ ). First, define a function $f^{u}(K, \theta)$ $=f(K, 1-\hat{l}(K, \theta), \theta)$ and consider the set of functions $\mathbf{M}$ for the inverse of marginal utility in equilibrium,

Definition: $\mathbf{M}_{l}=\{m(K, \theta) \mid m: \mathbf{K} \times \mathbf{\Theta} \rightarrow \mathbf{K}$ is continuous; $0 \leq$ $m(K, \theta) \leq \frac{1}{u_{c}\left(f^{u}(K, \theta), \hat{l}(K, \theta)\right)}$ for $K>0 ; m(K, \theta)=0$ for $K=0 ; \quad$ and $\frac{r\left(K^{\prime}, \theta\right)}{m\left(K^{\prime}, \theta\right)} \leq \frac{r(K, \theta)}{m(K, \theta)}$ for $\left.K^{\prime} \geq K\right\}$

By assumption D2, $r(K, \theta)$ is continuous and $\mathbf{K}$ is a compact set, therefore, $r$ is uniformly continuous. As in Section 4 , one can verify that $\mathbf{M}_{l}$ is a closed, equicontinuous, pointwise compact subset of the space of continuous functions on a compact topological space, namely $\mathbf{C}(\mathbf{S}) . \mathbf{M}_{l}$ is, therefore, compact. 
We now define a suitable operator on the space $\mathbf{M}_{l}$ and find a unique strictly positive fixed point of this operator (to prove the uniqueness of recursive equilibrium in $\overline{\mathbf{H}})$. As before, define the function $H(m, K, \theta)$ for each $m \in \mathbf{M}_{l}$ implicitly as follows (the following lemma makes sure that this definition is meaningful),

$$
\begin{aligned}
u_{c}(H(m(K, \theta), K, \theta), l(H(m(K, \theta), K, \theta), K, \theta)) & =\frac{1}{m(K, \theta)}, m>0 ; \\
\text { and } H(m, K, \theta) & =0, m=0 .
\end{aligned}
$$

Note that, $H(m(K, \theta), K, \theta)=h(K, \theta)$, pointwise. The proof of uniqueness takes place in three lemmata.

Lemma 35 Under assumptions P3, P4, T3 and D2, the mapping $H(m, K, \theta)$ is well-defined for each $m, K$ and $\theta$.

\section{Proof: Datta, Mirman and Reffett[22] Lemma 2.}

To characterize $H(m, K, \theta)$, take $m^{\prime} \geq m$ in the pointwise partial order on $\mathbf{M}_{l}$. Define $h_{2}=H\left(m^{\prime}, K, \theta\right)$ and $h_{1}=H(m, K, \theta)$. Notice when $m^{\prime} \geq m$, we have $h_{2} \geq h_{1}$. We can now show that $f(k, 1-l(H(m, K, \theta), K, \theta))-$ $H(m, K, \theta)$ is decreasing in $m$ by the definition of $H(m, K, \theta)$. Define

$$
\left.\Delta\left(h, f_{h}-h, \theta\right)=\beta \int u_{c}\left(h\left(f_{h}-h, \theta^{\prime}\right), l\left(h\left(f_{h}-h, \theta^{\prime}\right), f_{h}-h, \theta^{\prime}\right)\right) r\left(f_{h}-h, \theta^{\prime}\right) \chi\left(\theta, d \theta^{\prime}\right)\right)
$$

Then for $m^{\prime} \geq m$, we have the following inequality

$$
\begin{aligned}
u_{c}\left(A h_{1}, l\left(A h_{1}, K, \theta\right)\right. & =\Delta\left(h_{1}, f_{A h_{1}}-A h_{1}, \theta\right) \\
& \geq \Delta\left(h_{2}, f_{A h_{1}}-A h_{1}, \theta\right)
\end{aligned}
$$

Therefore, for such a perturbation of $h$, the mapping $Z$ used in the definition of $A h$ is now nonnegative. Therefore, the first term in the definition of $Z$ must decrease and the second term must increase in a solution $A h_{2}$. The latter implies $f_{A h_{2}}-A h_{2} \leq f_{A h_{1}}-A h_{1}$. Consequently, by the definition of $H(m, K, \theta), f(K, 1-l(H(m, K, \theta), K, \theta))-H(m, K, \theta)=f_{H(m)}-H(m)$ must be decreasing in $m$.

Now, define the mapping

$$
\hat{Z}(m, \tilde{m}, K, \theta)=\frac{1}{\tilde{m}}-\beta \int_{\Theta} \frac{r\left(f_{\tilde{m}}-H(\tilde{m}, K, \theta), \theta^{\prime}\right)}{m\left(f_{\tilde{m}}-H(\tilde{m}, K, \theta), \theta^{\prime}\right)} \chi\left(\theta, d \theta^{\prime}\right),
$$


where $f_{m}-H(m, K, \theta)=f(K, 1-l(H(m, K, \theta), K, \theta), \theta)-H(m, K, \theta)$ and we are ready to define the operator,

$$
\hat{A}(m)=\left\{\tilde{m} \in \mathbf{M}_{l} \mid \hat{Z}(m, \tilde{m}, K, \theta)=0 \text {, for } m>0 \text {; and, } 0 \text { elsewhere }\right\} .
$$

Defining the standard partial order on $\mathbf{M}_{l}$, that is, $m^{\prime} \geq m, m^{\prime}, m \in \mathbf{M}_{l}$ if and only if $m^{\prime}(K, \theta) \geq m(K, \theta)$ for all $(K, \theta)$. Finally, if $m^{\prime}(K, \theta)>$ $m(K, \theta), m, m^{\prime} \in \mathbf{M}_{l}$, the mapping $H$ must be such that $u_{c}(H(m, K, \theta), l(H(m, K, \theta), K, \theta))$ is decreasing in $m$ for each $(K, \theta)$. Since $h \in \overline{\mathbf{H}}, u_{c}(c, l(c, K, \theta)$ is decreasing in $c$, and there exists $h, h^{\prime} \in \overline{\mathbf{H}}$ such that $h^{\prime}=H\left(\frac{1}{u_{c}\left(h^{\prime}, l\left(h^{\prime}, K, \theta\right)\right)}, K, \theta\right)=$ $H\left(m^{\prime}, K, \theta\right)$ and $h=H\left(\frac{1}{u_{c}(h, l(h, K, \theta))}, K, \theta\right)=H(m, K, \theta)$.

If the operator $\hat{A} m$ is well defined, we are able to relate orbits of the operator $\hat{A}^{n} m_{0} \in \mathbf{M}_{l}$ to those of the operator $A^{n} h_{0} \in \overline{\mathbf{H}}$ by the following construction. Consider some $h_{0} \in \overline{\mathbf{H}}$. For such an $h_{0}$, there exists an $m_{0}=\frac{1}{u_{c}\left(h_{0}, l\left(h_{0}, K, \theta\right)\right)} \in \mathbf{M}_{l}$ such that $H\left(\frac{1}{u_{c}\left(h_{0}, l\left(h_{0}, K, \theta\right)\right)}\right)=h_{0}$. By definition, $\hat{Z}\left(m_{0}, \hat{A} m_{0}, K, \theta\right)=\hat{Z}\left(H\left(\frac{1}{u_{c}\left(h_{0}, l\left(h_{0}, K, \theta\right)\right)}, K, \theta\right), \hat{A} H\left(\frac{1}{u_{c}\left(A h_{0}, l\left(A h_{0}, K, \theta\right)\right)}\right), K, \theta\right)=$ $Z\left(h_{0}, A h_{0}, K, \theta\right)$.

Therefore, $h_{1}=A h_{0}=H\left(\frac{1}{u_{c}\left(A h_{0}, l\left(A h_{0}, K, \theta\right)\right)}\right)=H\left(\hat{A} m_{0}\right)$. A similar argument establishes $A^{n} h_{0}=H\left(\hat{A}^{n} m_{0}\right), n=1,2, \ldots$ We next show that the operator $\hat{A} m$ is well defined.

Lemma 36 Under assumptions P3, P4, T3 and D2, the operator $\hat{A}$ is a well-defined transformation on $\mathbf{M}_{l}$.

Proof: Datta et al[22] Lemma 3.

We now provide the last step of our argument.

Lemma 37 Under assumptions P3, P4, T3 and D2, if $\hat{A}$ has a strictly positive fixed point then it is unique.

Proof: Since $\hat{Z}$ is increasing in $m$, and decreasing in $\tilde{m}=\hat{A} m, \hat{A} m_{1} \geq$ $\hat{A} m_{2}$ for $m_{1} \geq m_{2}$. A sufficient condition for strong sublinearity is,

$$
\hat{Z}(t m, t \hat{A} m, K, \theta)>\hat{Z}(t m, \hat{A} t m, K, \theta) .
$$


This inequality follows since $m \in \mathbf{M}_{l}$, and $r$ decreasing in $K$. Thus,

$$
\hat{Z}(t m, t \hat{A} m, K, \theta)=\frac{1}{\tilde{m}}-\beta \int_{\Theta} \frac{r\left(f_{\tilde{m}}-H(t \tilde{m}), \theta^{\prime}\right)}{m\left(f_{\tilde{m}}-H(t \tilde{m}), \theta^{\prime}\right)} \chi\left(\theta, d \theta^{\prime}\right)>0,
$$

and $\hat{Z}(t m, \hat{A} t m, K, \theta)=0$. Therefore, by the same argument in Proposition 18, if $\hat{A}$ has a strictly positive fixed point, it is unique in $\mathbf{M}_{l}$ (and, therefore, in $\overline{\mathbf{H}})$.

Finally, we prove the existence a strictly positive fixed point.

Proposition 38 Under assumptions P3, P4, T3 and D2, there is a unique strictly positive MEDP.

Proof: Note that, as $\mathbf{M}_{l}$ is an order interval in a solid cone of continuous functions, and $\hat{A} m$ is strongly sublinear (see proof of lemma 37), by Guo and Lakshmikantham[35], Theorem 2.2.1.(a), $\hat{A}$ is e-concave on $\mathbf{M}_{l}$. As $\hat{A}$ is also isotone and $\mathbf{M}_{l}$ is also an order interval in a normal cone of continuous functions, by Guo and Lakshmikantham[35], Theorem 2.2.3, we conclude $\hat{A}$ is a cone compression. Then by the discussion in Guo and Lakshmikantham[35] (p65):An operator $\hat{A}$ is a cone compression if and only if $\hat{A}$ has a strictly positive fixed point. By lemma 37, we conclude that this strictly positive fixed point must be unique in $\mathbf{M}_{l}$. Finally, (noting the relationship between the orbits of $\hat{A}$ and $A$ discussed earlier in this section) as we have a unique strictly positive fixed point for $\hat{A}$, namely $m^{*}>0$, we must have a unique fixed point for $A$, say $h^{*}>0$. Since $h^{*}>0$ implies strictly positive consumption, it is a MEDP.

It is clear that Remark 19 also applies here. Therefore, the existence of a strictly positive fixed point is characterized by the operator $\hat{A}$ being a cone compression. Also, note that $h^{*}>0$ is crucial in Vailakis[79] for characterizing prices in $l_{+}^{1} \backslash\{0\}$.

\section{Concluding Remarks}

In this chapter, we survey a new and emerging approach to recursive competitive equilibrium theory that is commonly referred to as isotone recursive methods and we focus on homogenous agents' economies. These methods allow one to unify results on the existence, characterization and computation of MEDPs and the SME for a large class of economies commonly encountered in applied dynamic macroeconomics. Datta, Mirman, Morand and 
Reffett[23] develop isotone recursive methods to study MEDPs in the stochastic Ramsey models of Becker and Zilcha [10] with heterogeneous agents. They find sufficient conditions for MEDPs to be isotone and Lipschitz continuous and for MEDPs that are simply Lipschitz continuous. Another application of isotone recursive methods to the case of heterogeneous agent models is in overlapping generation models. These models form the basis of much work in lifecycle theory and the theory of social security. Erikson, Morand and Reffett[31] apply the isotone recursive approach to a class of two period stochastic lifecycle-overlapping generations models with social security, production nonconvexities and public policy (fiscal or monetary). Primarily, they consider the case of i. i. d. shocks but provide some preliminary results with Markov shock.

Potentially the most important extension of isotone recursive methods is the so-called "mixed-monotone" recursive methods first presented systematically in Reffett[64], and subsequently applied in Mirman, Reffett, and Stachurski[55] to Bewley models with a single asset. The mixed-monotone method build upon the mixed-monotone fixed point theory (also known as "coupled" fixed point theory) that has been developed in the literature on discontinuous differential equations. These methods appear powerful, and deliver MEDPs on the natural state space of current states even in situations where MEDPs are not unique. Discussions of mixed monotone fixed point theory are found in Amann[4], Heikkila and Lakshmikantham[36] and Reffett[63], to name a few. The discovery of mixed-monotone recursive methods appears to be a giant step forward in developing methods based on constructive fixed point theory that can be applied in a wide-array of economic situations. One no longer needs to have isotone operators (nor fixed point spaces) where underlying constructions are based on isotonicity. One problem with this method is that one requires sufficient topological structure relative to the fixed point space for antitone transformations to possess the fixed point property. Preliminary discussion in Reffett[62][63][64] indicates that for many interesting economies, such fixed point spaces are available. For example, these methods provide successive approximation algorithms for computing Bewley models of the sort studied in Aiyagari[2], Krusell and Smith[47], and Miao[52]. In addition, isotone recursive methods are a special case of mixed monotone recursive methods and can be studied in a "single" step using an isotone operators instead of multi-steps for mixed-monotone operators. Mixed monotone recursive methods unify the existing approaches to characterize MEDPs and the SME by allowing researchers to obtain more general results that relate monotone iterative computational procedures to actual fixed point constructions. As numerical 
methods described in standard monographs (e.g., Krasnoselskii et al[44]) can build on explicit operators to obtain error estimates of Santos and Vigo[70] and Santos[69]. In principle, one might be able to obtain a complete set of iterative methods for studying numerically, the quantitative properties of the SME in a large class of macroeconomic models to a specified degree of accuracy, which seems to be the goal of quantitative macroeconomics (e.g., real business cycle studies). Indeed, qualitative methods can provide an essential, first step in obtaining a useful (and, mathematically credible) quantitative theory of macroeconomic fluctuations and long-run growth.

\section{References}

[1] Abian, S. and A.B. Brown. 1962. A theorem on partially order sets with applications to fixed point theorems. Canadian Journal of Mathematics, $13,78-82$.

[2] Aiyagari, R. 1994. Uninsured idiosyncratic risk and aggregate saving. Quarterly Journal of Economics, 109, 659-684.

[3] Amann, H. 1976. Fixed equations and nonlinear eigenvalue problems in ordered Banach spaces. SIAM Review, 18(4), 620-709.

[4] Amann, H. 1977. Order Structures and Fixed Points.SAFA 2, ATTI del 2。 Seminario di Analisi Funzionale e Applicazioni. MS.

[5] Amir, R. 1996. Sensitivity analysis of multisector optimal economic dynamics, Journal of Mathematical Economics, 25, 123-141.

[6] Amir, R. L. Mirman, and W. Perkins. 1991, One-sector nonclassical optimal growth: optimality conditions and comparative dynamics. International Economic Review, 32, 625-644.

[7] Antoniadou, E. 1995. Lattice Programming and Economic Optimization. Ph.D. Dissertation. Stanford University.

[8] Askri, and C. LeVan. 1998. Differentiability of the value function of nonclassical optimal growth models. Journal of Optimization Theory and Applications. 97 (3), 591-604.

[9] Becker, R. and C. Foias. 1998. Implicit programming and the invariant manifold for Ramsey equilibria. in Y. Abramovich, E. Avgerinos, and N. Yannelis, eds. Functional Analysis and Economic Theory, 1998, Springer-Verlag. 
[10] Becker R, and I. Zilcha. 1997. Stationary Ramsey equilibria under uncertainty. Journal of Economic Theory, 75, 122-140.

[11] Berge, C. 1963. Topological Spaces, MacMillan Press.

[12] Bewley, T. 1986. Stationary monetary equilibrium with a continuum of independently fluctuating consumers. in Contributions to Mathematics in Honor of Gerard Debreu, ed. W. Hildenbrand and A. Mas-Colell. North-Holland, Amsterdam.

[13] Birkhoff, G. 1967. Lattice Theory. AMS Press.

[14] Bizer, D. and K. Judd. 1989. Taxation and uncertainty. American Economic Review, 79, 331-336.

[15] Brock, W. and L. Mirman. 1972. Optimal growth and uncertainty: the discounted case. Journal of Economic Theory, 4, 479-513.

[16] Bourbaki, N. 1950, Sur le Théorème de Zorn, Archiv der Mathematik, 2 (1949-1950), 434-437.

[17] Clarke, F. 1983. Optimization and Nonsmooth Analysis. SIAM Press.

[18] Coleman, W.J. II. 1990. Solving the stochastic growth model by policyfunction iteration. Journal of Business and Economic Statistics, 8, 2729 .

[19] Coleman, W.J., II. 1991. Equilibrium in a production economy with an income tax. Econometrica, 59, 1091-1104.

[20] Coleman, W.J., II. 1997. Equilibria in distorted infinite-horizon economies with capital and labor, Journal of Economic Theory, 72 , 446-461.

[21] Coleman, W. J., II. 2000. Uniqueness of an equilibrium in infinitehorizon economies subject to taxes and externalities, Journal of Economic Theory 95, 71-78.

[22] Datta, M., L. Mirman and K. Reffett. 2002. Existence and uniqueness of equilibrium in distorted dynamic economies with capital and labor Journal of Economic Theory, 103, 377-410.

[23] Datta, M., L. Mirman, O. Morand and K. Reffett. 2005. Markovian Equilibrium in Infinite Horizon Economies with Many Agents, Incomplete Markets and Public Policy. Journal of Mathematical Economics, forthcoming. 
[24] Davey, B. and H. Priestley. 2002. Introduction to Lattices and Order. Cambridge Press, 2nd edition.

[25] Davis, A. 1955. A characterization of complete lattices. Pacific Journal of Mathematics, 5, 311-319.

[26] Debreu, G. 1967. Integration of correspondences. Proceedings of the Fifth Berkeley Symposium on Mathematics, Statistics, and Probability, II, Part 1, eds. L. LeCam, J. Neyman, and E.L. Scott. University of California Press. 351-372.

[27] Dieudonne, J. 1960. Foundations of Modern Analysis. Academic Press.

[28] Donaldson, J. and R. Mehra. 1983. Stochastic growth with correlated production shocks. Journal of Economic Theory, 29, 282-312.

[29] Dudley, R. M. 1989. Real Analysis and Probability, Wadsworth.

[30] Dugundji, J and V. Granas. 1982. Fixed Point Theory, Polish Scientific Press.

[31] Erikson, J., O. Morand, and K. Reffett. 2004. Isotone Recursive Methods for Overlapping Generations Models. MS. Arizona State University.

[32] Frink, O. 1942. Topology in lattices. Transactions of the American Mathematical Society, 51, 569-582.

[33] Gauvin, J. and F. Dubeau. 1982. Differential properties of the marginal function in mathematical programming. Mathematical Programming Studies, 19, 101-119.

[34] Greenwood, J., and G. Huffman. 1995. On the existence of nonoptimal equilibria in dynamic stochastic economies, Journal of Economic Theory, 65, 611-623.

[35] Guo, D. and V. Lakshmikantham. 1988. Nonlinear Problems in Abstract Cones. Academic Press.

[36] Heikkila, S. and V. Lakshmikantham. 1994. Monotone iterative techniques for discontinuous nonlinear differential equations, Marcel Dekker.

[37] Heikkila, S. and K. Reffett. 2004. Fixed point theorems for multivalued Functions. MS. Mathematics-University of Oulu. 
[38] Heikkila, S. and S. Hu. 1993. On fixed points of multifunctions in ordered spaces. Applicable Analysis, 51, 115-127.

[39] Hopenhayn, H. and E. Prescott. 1992. Stochastic monotonicity and stationary distributions for dynamic economies. Econometrica, 60, 13871406.

[40] Jachymski, J. 2001. Order-theoretic aspects of metric fixed point theory. in Handbook of Metric Fixed Point Theory, ed. W.A. Kirk and B. Sims, Kluwer. 613-641.

[41] Jachymski, J. 2003. Converses to fixed point theorems of Zermelo and Caristi. Nonlinear Analysis, 52, 1455-63.

[42] Judd, K. 1992. Projection methods for solving aggregate growth models. Journal of Economic Theory, 58, 410-452.

[43] Kantorovich, L. The method of successive approximation for functional equations. 1939. Acta Math. 71, 63-97.

[44] Krasnoselskii, MA, G.M. Vainikko, P.P. Zabreiko, Ya.B. Rutitiskii, V. Ya. Stetsenko, 1972. Approximate solution of Operator Equations. Wolters-Noordhoff Press.

[45] Krebs, T. 2004. Non-existence of recursive equilibria on compact state spaces when markets are incomplete. Journal of Economic Theory, 115, 134-150.

[46] Krebs, T. 2004. Recursive equilibrium in endogenous growth models with incomplete markets. MS. Brown University.

[47] Krusell, P. and A. Smith. 1998. Income and wealth heterogeneity in the macroeconomy, Journal of Political Economy, 106, 867-896.

[48] LeVan, C. and L. Morhaim. Optimal growth models with bounded or unbounded returns: a unifying approach. Journal of Economic Theory, 105(1), 158-18\%.

[49] Li Calzi, M. and A. Veinott, Jr. 1991. Subextremal functions and lattice programming. MS. Stanford University.

[50] Lovejoy, W. 1987. Ordered solutions for dynamic programs. Mathematics of Operations Research, 269-278. 
[51] Lucas, R. E., Jr. and N. Stokey. 1987. Money and interest in a cash-inadvance economy. Econometrica, 55, 1821-37.

[52] Miao, J. 2003. Competitive equilibria in economies with a continuum of consumer and aggregate shocks. MS. University of Rochester.

[53] Milgrom, P. and C. Shannon. 1994. Monotone comparative statics. Econometrica, 62, 157-180.

[54] Mirman, L., O. Morand, and K. Reffett. 2004. A qualitative approach to Markovian equilibrium in infinite horizon economies with capital. MS. Arizona State University.

[55] Mirman, L., K. Reffett, and J. Stachurski. 2004. Computing Bewley models. MS. Arizona State University.

[56] Mirman, L. and I. Zilcha. 1975. On optimal growth under uncertainty. Journal of Economic Theory, 11, 329-339.

[57] Morand, O. and K. Reffett. 2003. Existence and uniqueness of equilibrium in nonoptimal unbounded infinite horizon economies. Journal of Monetary Economics. 50, 1351-1373.

[58] Morand, O. and K. Reffett. 2004. Monotone Map Methods for Overlapping Generations Models with nonclassical technologies: The Case of Markov Shocks. MS. Arizona State University.

[59] Muenzenberger, T. and R. Smithson. 1973. Fixed point structures. American Mathematical Society, 184, 153-173.

[60] Pelczar, A. 1961 On the invariant points of a transformation. Ann. Pol Math, 11, 199-202.

[61] Prescott, E. and R. Mehra. 1980. Recursive competitive equilibrium: the case of homogeneous households. Econometrica, 48:1365-1379

[62] Reffett, K. 2004. Ordered Markovian equilibrium. MS, Arizona State University.

[63] Reffett, K. 2004. Mixed monotone fixed point methods with economic applications. MS. Arizona State University.

[64] Reffett, K. 2004. Mixed monotone recursive methods. MS. Arizona State University. 
[65] Rockafellar, R.T. 1980. Generalized directional derivatives and subgradients of nonconvex functions. Canadian Journal of Mathematics, $32(2), 257-280$.

[66] Rockafellar, R.T. and R. Wets. Variational Analysis. Springer Verlag.

[67] Santos, M. 1991. Smoothness of the policy function in discrete-time models. Econometrica, 1365-1382.

[68] Santos, M. 2002. On non existence of Markov equilibria in competitivemarket economies." Journal of Economic Theory, 105, 73-98.

[69] Santos, M. 2000. The numerical accuracy of numerical solutions using Euler residuals. Econometrica, 68, 1377-140.

[70] Santos, M. and J. Vigo-Aguiar. 1998. Analysis of a numerical dynamic programming algorithm applied to economic models. Econometrica, 66, 409-426.

[71] Shannon, C. 1995. Weak and strong monotone comparative statics. Economic Theory, 5, 209-237.

[72] Smithson, R. 1971. Fixed points of order preserving multifunctions. Proceedings of the American Mathematical Society, 28(1), 304-310.

[73] Stokey, N., R.E. Lucas, Jr., with E. Prescott. 1989. Recursive methods in economic dynamics. Harvard Press

[74] Tarski, A. A fixed point for lattices and its applications. Bull. of Amer. Math. Soc. 55, 1051-52.

[75] Tarski, A. 1955. A lattice-theoretical fixpoint theorem and its applications. Pacific Journal of Mathematics, 5, 285-309.

[76] Topkis, D. 1978. Minimizing a submodular function on a lattice. Operations Research, 26, 305-321.

[77] Topkis, D. 1979. Equilibrium points in nonzero sum $n$-person submodular games. SIAM Journal of Control and Optimization, 17, 773-787.

[78] Topkis, D. 1998. Supermodularity and Complementarity. Princeton Press.

[79] Vailakis, Y. 2004. Simple proof of existence of equilibrium in a single sector growth model with elastic labor supply. CERMSEM U of Paris Working Paper. 
[80] Veinott, A. 1989. Lattice Programming, Notes Johns Hopkins University. MS.

[81] Veinott, A. 1992. Lattice programming: qualitative optimization and equilibria. MS. Stanford

[82] Vulikh, B. 1967. Introduction to the Theory of Partially Ordered Spaces, Noordhoff Scientific Publishers.

[83] Zhou, L. 1994. The set of Nash equilibria of a supermodular game is a complete lattice. Games and Economic Behavior, 7, 295-300.

[84] Zeidler, E. 1986. Nonlinear Functional Analysis and its Applications, vol1. Springer Verlag. 